\title{
Logarithmic Hybrid Aggregation Operators Based on Single Valued Neutrosophic Sets and Their Applications in Decision Support Systems
}

\author{
Shahzaib Ashraf ${ }^{1}\left(\mathbb{D}\right.$, Saleem Abdullah ${ }^{1, *}$, Florentin Smarandache ${ }^{2}$ and Noor ul Amin ${ }^{3}$ \\ 1 Department of Mathematics, Abdul Wali Khan Uniersity, Mardan 23200, Pakistan; \\ shahzaibashraf@awkum.edu.pk \\ 2 Department of Mathematics and Sciences, University of New Mexico, 705 Gurley Ave., \\ Gallup, NM 87301, USA; fsmarandache@gmail.com \\ 3 Department of Information Technology, Hazara University, Mansehra 21300, Pakistan; namin@hu.edu.pk \\ * Correspondence: saleemabdullah@awkum.edu.pk
}

Received: 17 January 2019; Accepted: 7 March 2019; Published: 11 March 2019

check for updates

\begin{abstract}
Recently, neutrosophic sets are found to be more general and useful to express incomplete, indeterminate and inconsistent information. The purpose of this paper is to introduce new aggregation operators based on logarithmic operations and to develop a multi-criteria decision-making approach to study the interaction between the input argument under the single valued neutrosophic (SVN) environment. The main advantage of the proposed operator is that it can deal with the situations of the positive interaction, negative interaction or non-interaction among the criteria, during decision-making process. In this paper, we also defined some logarithmic operational rules on SVN sets, then we propose the single valued neutrosophic hybrid aggregation operators as a tool for multi-criteria decision-making (MCDM) under the neutrosophic environment and discussd some properties. Finally, the detailed decision-making steps for the single valued neutrosophic MCDM problems were developed, and a practical case was given to check the created approach and to illustrate its validity and superiority. Besides this, a systematic comparison analysis with other existent methods is conducted to reveal the advantages of our proposed method. Results indicate that the proposed method is suitable and effective for decision process to evaluate their best alternative.
\end{abstract}

Keywords: single valued neutrosophic sets; logarithmic operational laws; logarithmic aggregation operators; MCGDM problems

\section{Introduction}

The information involves, in most of the real-life decision-making problems are often incomplete, indeterminate and inconsistent. Fuzzy set theory introduced by Zadeh [1] deals with imprecise, inconsistent information. Although fuzzy set information proved to be very handy but it cannot express the information about rejection. Atanassov [2] introduced the intuitionistic fuzzy set (IFS) to bring in non-membership. Non membership function represents degree of rejection. To incorporate indeterminate and inconsistent information, in addition to incomplete information, the concept of neutrosophic set (NS) proposed by Smarandache [3]. A NS generalizes the notion of the classic set, fuzzy set (FS) [1], IFS [2], paraconsistent set [4], dialetheist set, paradoxist set [4], and tautological set [4] to name a few. In NS, indeterminacy is quantified explicitly, and truth, indeterminacy, and falsity memberships are expressed independently. The NS generalizes different types of non-crisp sets but in real scientific and engineering applications the NS and the set-theoretic operators require to be specified. For a detailed study on NS we refer to [5-17]. 


\section{Related Work}

Most of the weighted aggregation operators consider situations in which criteria and preferences of experts are independent, which means that additivity is a main property of these operators. However, in real life decision-making problems, the criteria of the problems are often interdependent or interactive.

Most of the weighted average operators are based on the basic algebraic product and algebraic sum of single valued neutrosophic numbers (SVNNs) which are not the only operations available to model the intersection and union of SVNNs. The logarithmic algebraic product and sum are two good alternatives of algebraic operations which can be used the model intersection and union of SVNNs. Moreover, it is observed that in the literature there is little investigation on aggregation operators utilizing the logarithmic operations on SVNNs. For a detailed review on the applications of logarithmic operations, we refer to [10]. As already mentioned that the single valued neutrosophic set (SVNS) is an effective tool to describe the uncertain, incomplete and indeterminate information. The logarithmic single valued neutrosophic hybrid and logarithmic generalized single valued neutrosophic algebraic operators have the ability to express interactions among the criteria and it can replace the weighted average to aggregate dependent criteria for obtaining more accurate results. Motivated by these, we find it interesting to develop the logarithmic single valued neutrosophic hybrid aggregation operators for decision-making with neutrosophic information.

Also, we proposed the possibility of a degree-ranking technique for SVNNs from the probability point of view, since the ranking of SVNNs is very important for decision-making under the SVN environment. Furthermore, we proposed a multi-criteria decision-making model based on the logarithmic single valued neutrosophic hybrid weighted operators. Forstudy the multi-criteria decision-making models, we refer [18-31].

The aim of writing this paper is to introduce a decision-making method for MCDM problems in which there exist interrelationships among the criteria. The contributions of this research are:

(1) A novel logarithmic operations for neutrosophic information is defined, which can overcome the weaknesses of algebraic operations and obtain the relationship between various SVNNs.

(2) Logarithmic operators for IFSs are extended to logarithmic single-valued neutrosophic hybrid operators and logarithmic generalized single-valued neutrosophic operators, namely, logarithmic single valued neutrosophic hybrid weighted averaging (L-SVNHWA), logarithmic single valued neutrosophic hybrid weighted geometric (L-SVNHWG), logarithmic generalized single-valued neutrosophic weighted averaging (L-GSVNWA) and logarithmic single-valued neutrosophic weighted geometric (L-GSVNWG) to SVNSs, which can overcome the algebraic operators drawbacks.

(3) A decision-making approach to handle the MCDM problems under the neutrosophic informations is introduced.

To attain our research goals which are stated above, the arrangement of the paper is offered as: Section 2 concentrates on basic definitions and operations of existing extensions of fuzzy set theories. In Section 3, some novel logarithmic operational laws of SVNSs are presented. Section 4 defines the logarithmic hybrid aggregation operators for SVNNs. In Section 5, an algorithm for handling the neutrosophic MCDM problem based on the developed logarithmic operators is presented. In Section 5.1, an application to verify the novel method is given and Section 5.2 presents the comparison study about algebraic and logarithmic aggregation operators. Section 6 consists of the conclusion of the study.

\section{Preliminaries}

This section includes the concepts and basic operations of existing extensions of fuzzy sets to make the study self contained. 
Definition 1. [2] For a set $\Re$, by an intuitionistic fuzzy set in $\Re$, we have a structure

$$
\zeta=\left\{\left\langle P_{\sigma}(r), N_{\sigma}(r)\right\rangle \mid r \in \Re\right\},
$$

in which $P_{\sigma}: \Re \rightarrow \Theta$ and $N_{\sigma}: \Re \rightarrow \Theta$ indicate the membership and non-membership grades in $\Re, \Theta=[0,1]$ be the unit interval. Also the following condition is satisfied by $P_{\sigma}$ and $N_{\sigma}, 0 \leq P_{\sigma}(r)+N_{\sigma}(r) \leq 1 ; \forall r \in \Re$. Then $\zeta$ is said to be intuitionistic fuzzy set in $\Re$.

Definition 2. [32] For a set $\Re$, by a neutrosophic set in $\Re$, we have a structure

$$
\zeta=\left\{\left\langle P_{\sigma}(r), I_{\sigma}(r), N_{\sigma}(r)\right\rangle \mid r \in \Re\right\},
$$

in which $P_{\sigma}: \Re \rightarrow \Theta, I_{\sigma}: \Re \rightarrow \Theta$ and $N_{\sigma}: \Re \rightarrow \Theta$ indicate the truth, indeterminacy and falsity memberships in $\Re, \Theta=] 0^{-}, 1^{+}$. Also the following condition is satisfied by $P_{\sigma}, I_{\sigma}$ and $N_{\sigma}, 0^{-} \leq P_{\sigma}(r)+I_{\sigma}(r)+N_{\sigma}(r) \leq$ $3^{+} ; \forall r \in \Re$. Then, $\zeta$ is said to be neutrosophic set in $\Re$.

Definition 3. [33] For a set $\Re$, by a single valued neutrosophic set in $\Re$, we mean a structure

$$
\zeta=\left\{\left\langle P_{\sigma}(r), I_{\sigma}(r), N_{\sigma}(r)\right\rangle \mid r \in \Re\right\},
$$

in which $P_{\sigma}: \Re \rightarrow \Theta, I_{\sigma}: \Re \rightarrow \Theta$ and $N_{\sigma}: \Re \rightarrow \Theta$ indicate the truth, indeterminacy and falsity memberships in $\Re, \Theta=[0,1]$. Also the following condition is satisfied by $P_{\sigma}, I_{\sigma}$ and $N_{\sigma}, 0 \leq P_{\sigma}(r)+I_{\sigma}(r)+$ $N_{\sigma}(r) \leq 3 ; \forall r \in \Re$. Then, $\zeta$ is said to be a single valued neutrosophic set in $\Re$. We denote this triplet $\zeta=\left\langle P_{\sigma}(r), I_{\sigma}(r), N_{\sigma}(r)\right\rangle$, in whole study called SVNN.

Ye [14], Wang et al. [33] and [34] proposed the basic operations of SVNNs, which are as follows:

Definition 4. [34] For any two SVNNs $\zeta_{p}=\left\langle P_{\xi_{p}}(r), I_{\xi_{p}}(r), N_{\xi_{p}}(r)\right\rangle$ and $\zeta_{q}=$ $\left\langle P_{\xi_{q}}(r), I_{\xi_{q}}(r), N_{\xi_{q}}(r)\right\rangle$ in $\Re$. The union, intersection and compliment are proposed as:

(1) $\zeta_{p} \subseteq \zeta_{q} \quad$ iff $\quad \forall r \in \Re, P_{\xi_{p}}(r) \leq P_{\xi_{q}}(r), I_{\xi_{p}}(r) \geq I_{\xi_{q}}(r)$ and $N_{\xi_{p}}(r) \geq N_{\xi_{q}}(r)$;

(2) $\zeta_{p}=\zeta_{q}$ iff $\zeta_{p} \subseteq \zeta_{q}$ and $\zeta_{q} \subseteq \zeta_{p}$;

(3) $\zeta_{p} \cup \zeta_{q}=\left\langle\max \left(P_{\xi_{p}}, P_{\xi_{q}}\right), \min \left(I_{\xi_{p}}, I_{\xi_{q}}\right), \min \left(N_{\xi_{p}}, N_{\xi_{q}}\right)\right\rangle$;

(4) $\zeta_{p} \cap \zeta_{q}=\left\langle\min \left(P_{\xi_{p}}, P_{\xi_{q}}\right), \max \left(I_{\xi_{p}}, I_{\xi_{q}}\right), \max \left(N_{\xi_{p}}, N_{\xi_{q}}\right)\right\rangle$;

(5) $\zeta_{p}^{c}=\left\langle N_{\xi_{p}}, I_{\xi_{p}}, P_{\xi_{p}}\right\rangle$.

Definition 5. $[13,15,33]$ For any two SVNNs $\zeta_{p}=\left\langle P_{\xi_{p}}(r), I_{\xi_{p}}(r), N_{\xi_{p}}(r)\right\rangle$ and $\zeta_{q}=$ $\left\langle P_{\xi_{q}}(r), I_{\xi_{q}}(r), N_{\xi_{q}}(r)\right\rangle$ in $\Re$ and $\beta \geq 0$.Then the operations of $S V N N$ s are proposed as:

(1) $\zeta_{p} \oplus \zeta_{q}=\left\{P_{\xi_{p}}+P_{\xi_{q}}-P_{\xi_{p}} \cdot P_{\xi_{q}}, I_{\xi_{p}} \cdot I_{\xi_{q}}, N_{\xi_{p}} \cdot N_{\xi_{q}}\right\}$;

(2) $\beta \cdot \zeta_{p}=\left\{1-\left(1-P_{\xi_{p}}\right)^{\beta},\left(I_{\xi_{p}}\right)^{\beta},\left(N_{\xi_{p}}\right)^{\beta}\right\}$;

(3) $\zeta_{p} \otimes \zeta_{q}=\left\{P_{\xi_{p}} \cdot P_{\xi_{q}}, I_{\xi_{p}}+I_{\xi_{q}}-I_{\xi_{p}} \cdot I_{\xi_{q}}, N_{\xi_{p}}+N_{\xi_{q}}-N_{\xi_{p}} \cdot N_{\xi_{q}}\right\}$;

(4) $\zeta_{p}^{\beta}=\left\{\left(P_{\xi_{p}}\right)^{\beta}, 1-\left(1-I_{\xi_{p}}\right)^{\beta}, 1-\left(1-N_{\xi_{p}}\right)^{\beta}\right\}$.

(5) $\beta^{\zeta_{p}}=\left\{\begin{array}{cc}\left(\beta^{1-P_{\tilde{\xi} p}}, 1-\beta^{I_{\tilde{\xi} p}}, 1-\beta^{N_{\tilde{\xi} p}}\right) & \text { if } \beta \in(0,1) \\ \left(\left(\frac{1}{\beta}\right)^{1-P_{\tilde{\xi} p}}, 1-\left(\frac{1}{\beta}\right)^{I_{\tilde{\xi} p}}, 1-\left(\frac{1}{\beta}\right)^{N_{\tilde{\xi} p}}\right) & \text { if } \beta \geq 1\end{array}\right.$

Definition 6. [33] For any three SVNNs $\zeta_{p}=\left\langle P_{\xi_{p}}(r), I_{\xi_{p}}(r), N_{\xi_{p}}(r)\right\rangle, \zeta_{q}=\left\langle P_{\xi_{q}}(r), I_{\xi_{q}}(r), N_{\xi_{q}}(r)\right\rangle$ and $\zeta_{l}=\left\langle P_{\sigma_{l}}(r), I_{\sigma_{l}}(r), N_{\sigma_{l}}(r)\right\rangle$ in $\Re$ and $\beta_{1}, \beta_{2} \geq 0$. Then, we have 
(1) $\zeta_{p} \oplus \zeta_{q}=\zeta_{q} \oplus \zeta_{p} ;$

(2) $\zeta_{p} \otimes \zeta_{q}=\zeta_{q} \otimes \zeta_{p}$

(3) $\beta_{1}\left(\zeta_{p} \oplus \zeta_{q}\right)=\beta_{1} \zeta_{p} \oplus \beta_{1} \zeta_{q}, \beta_{1}>0$;

(4) $\left(\zeta_{p} \otimes \zeta_{q}\right)^{\beta_{1}}=\zeta_{p}^{\beta_{1}} \otimes \zeta_{q}^{\beta_{1}}, \beta_{1}>0$;

(5) $\beta_{1} \zeta_{p} \oplus \beta_{2} \zeta_{p}=\left(\beta_{1}+\beta_{2}\right) \zeta_{p}, \beta_{1}>0, \beta_{2}>0$;

(6) $\zeta_{p}^{\beta_{1}} \otimes \zeta_{p}^{\beta_{2}}=\zeta_{p}^{\beta_{1}+\beta_{2}}, \beta_{1}>0, \beta_{2}>0$;

(7) $\left(\zeta_{p} \oplus \zeta_{q}\right) \oplus \zeta_{l}=\zeta_{p} \oplus\left(\zeta_{q} \oplus \zeta_{l}\right)$;

(8) $\left(\zeta_{p} \otimes \zeta_{q}\right) \otimes \zeta_{l}=\zeta_{p} \otimes\left(\zeta_{q} \otimes \zeta_{l}\right)$.

Definition 7. [33] For any $S V N N \zeta_{p}=\left\langle P_{\xi_{p}}(r), I_{\xi_{p}}(r), N_{\xi_{p}}(r)\right\rangle$ in $\Re$. Then score and accuracy values are defined as:

(1) $\widetilde{S}\left(\zeta_{p}\right)=P_{\xi_{p}}-I_{\xi_{p}}-N_{\xi_{p}}$

(2) $\widetilde{A}\left(\zeta_{p}\right)=P_{\xi_{p}}+I_{\xi_{p}}+N_{\xi_{p}}$

The above definitions of score and accuracy funtions suggest which SVNN is greater than other SVNNs. The comparison technique is defined in following definition.

Definition 8. [33] For any $S V N N s \zeta_{p}=\left\langle P_{\xi_{p}}(r), I_{\xi_{p}}(r), N_{\xi_{p}}(r)\right\rangle(p=1,2)$ in $\Re$.

Then comparison techniques are proposed as:

(1) If $\widetilde{S}\left(\zeta_{1}\right)<\widetilde{S}\left(\zeta_{2}\right)$, then $\zeta_{1}<\zeta_{2}$,

(2) If $\widetilde{S}\left(\zeta_{1}\right)>\widetilde{S}\left(\zeta_{2}\right)$, then $\zeta_{1}>\zeta_{2}$,

(3) If $\widetilde{S}\left(\zeta_{1}\right)=\widetilde{S}\left(\zeta_{2}\right)$, and

(a) $\widetilde{A}\left(\zeta_{1}\right)<\widetilde{A}\left(\zeta_{2}\right)$, then $\zeta_{1}<\zeta_{2}$,

(b) $\widetilde{A}\left(\zeta_{1}\right)>\widetilde{A}\left(\zeta_{2}\right)$, then $\zeta_{1}>\zeta_{2}$,

(c) $\widetilde{A}\left(\zeta_{1}\right)=\widetilde{A}\left(\zeta_{2}\right)$, then $\zeta_{1} \approx \zeta_{2}$.

Garg and Nancy [10] proposed some logarithmic-based aggregation operators, which are as follows:

Definition 9. [10] For any collection of SVNNs $\zeta_{p}=\left\langle P_{\xi_{p}}(r), I_{\xi_{p}}(r), N_{\xi_{p}}(r)\right\rangle(p=1,2, \ldots, n)$ in $\Re$, with $0<\sigma_{p} \leq \min \left\{P_{\xi_{p}}, 1-I_{\xi_{p}}, 1-N_{\xi_{p}}\right\}<1, \sigma \neq 1$. Then, the structure of logarithmic single valued neutrosophic weighted averaging (L-SVNWA) operator is defined as:

$$
L-\operatorname{SVNWA}\left(\zeta_{1}, \zeta_{2}, \ldots, \zeta_{n}\right)=\left(\begin{array}{c}
1-\prod_{p=1}^{n}\left(\log _{\sigma_{p}} P_{\xi_{p}}\right)^{\beta_{p}}, \\
\prod_{p=1}^{n}\left(\log _{\sigma_{p}}\left(1-I_{\xi_{p}}\right)\right)^{\beta_{p}}, \\
\prod_{p=1}^{n}\left(\log _{\sigma_{p}}\left(1-N_{\xi_{p}}\right)\right)^{\beta_{p}}
\end{array}\right),
$$

where $\beta_{p}(p=1,2, \ldots, n)$ are weight vectors with $\beta_{p} \geq 0$ and $\sum_{p=1}^{n} \beta_{p}=1$. 
Definition 10. [10] For any collection of $S V N N s \zeta_{p}=\left\langle P_{\xi_{p}}(r), I_{\xi_{p}}(r), N_{\xi_{p}}(r)\right\rangle(p=1,2, \ldots, n)$ in $\Re$, with $0<\sigma_{p} \leq \min \left\{P_{\xi_{p}}, 1-I_{\xi_{p}}, 1-N_{\xi_{p}}\right\}<1, \sigma \neq 1$. Then, the structure of the logarithmic single-valued neutrosophic-ordered weighted averaging (L-SVNOWA) operator is defined as:

$$
L-\operatorname{SVNOWA}\left(\zeta_{1}, \zeta_{2}, \ldots, \zeta_{n}\right)=\left(\begin{array}{c}
1-\prod_{p=1}^{n}\left(\log _{\sigma_{p}} P_{\zeta_{\eta(p)}}\right)^{\beta_{p}}, \\
\prod_{p=1}^{n}\left(\log _{\sigma_{p}}\left(1-I_{\zeta_{\eta(p)}}\right)\right)^{\beta_{p}}, \\
\prod_{p=1}^{n}\left(\log _{\sigma_{p}}\left(1-N_{\zeta_{\eta(p)}}\right)\right)^{\beta_{p}}
\end{array}\right),
$$

where $\beta_{p}(p=1,2, \ldots, n)$ are weighting vector with $\beta_{p} \geq 0, \sum_{p=1}^{n} \beta_{p}=1$ and pth largest weighted value is $\zeta_{\eta(p)}$ consequently by total order $\zeta_{\eta(1)} \geq \zeta_{\eta(2)} \geq \ldots \geq \zeta_{\eta(n)}$.

Definition 11. [10] For any collection of SVNNs $\zeta_{p}=\left\langle P_{\xi_{p}}(r), I_{\xi_{p}}(r), N_{\xi_{p}}(r)\right\rangle(p=1,2, \ldots, n)$ in $\Re$, with $0<\sigma_{p} \leq \min \left\{P_{\xi_{p}}, 1-I_{\xi_{p}}, 1-N_{\xi_{p}}\right\}<1, \sigma \neq 1$. Then, the structure of logarithmic single-valued neutrosophic-weighted geometric (L-SVNWG) operator is defined as:

$$
L-\operatorname{SVNWG}\left(\zeta_{1}, \zeta_{2}, \ldots, \zeta_{n}\right)=\left(\begin{array}{c}
\prod_{p=1}^{n}\left(1-\log _{\sigma_{p}} P_{\xi_{p}}\right)^{\beta_{p}}, \\
1-\prod_{p=1}^{n}\left(1-\log _{\sigma_{p}}\left(1-I_{\xi_{p}}\right)\right)^{\beta_{p}}, \\
1-\prod_{p=1}^{n}\left(1-\log _{\sigma_{p}}\left(1-N_{\xi_{p}}\right)\right)^{\beta_{p}}
\end{array}\right),
$$

where $\beta_{p}(p=1,2, \ldots, n)$ are weight vectors with $\beta_{p} \geq 0$ and $\sum_{p=1}^{n} \beta_{p}=1$.

Definition 12. [10] For any collection of SVNNs $\zeta_{p}=\left\langle P_{\xi_{p}}(r), I_{\tilde{\xi} p}(r), N_{\xi_{p}}(r)\right\rangle(p=1,2, \ldots, n)$ in $\Re$, with $0<\sigma_{p} \leq \min \left\{P_{\xi_{p}}, 1-I_{\xi_{p}}, 1-N_{\xi_{p}}\right\}<1, \sigma \neq 1$. Then, the structure of logarithmic single valued neutrosophic ordered weighted geometric (L-SVNOWG) operator is defined as:

$$
L-\operatorname{SVNOWG}\left(\zeta_{1}, \zeta_{2}, \ldots, \zeta_{n}\right)=\left(\begin{array}{c}
\prod_{p=1}^{n}\left(1-\log _{\sigma_{p}} P_{\xi_{\eta(p)}}\right)^{\beta_{p}}, \\
1-\prod_{p=1}^{n}\left(1-\log _{\sigma_{p}}\left(1-I_{\xi_{\eta(p)}}\right)\right)^{\beta_{p}}, \\
1-\prod_{p=1}^{n}\left(1-\log _{\sigma_{p}}\left(1-N_{\xi_{\eta(p)}}\right)\right)^{\beta_{p}}
\end{array}\right),
$$

where $\beta_{p}(p=1,2, \ldots, n)$ are weighting vector with $\beta_{p} \geq 0$ and $\sum_{p=1}^{n} \beta_{p}=1$ and $p$ th are the largest weighted value is $\zeta_{\eta(p)}$ consequently by total order $\zeta_{\eta(1)} \geq \zeta_{\eta(2)} \geq \ldots \geq \zeta_{\eta(n)}$.

\section{Logarithmic Operational Laws}

Motivated by the well growing concept of SVNSs, we introduce some novel logarithmic operational laws for single valued neutrosophic numbers. As in real number systems $\log _{\sigma} 0$ is meaningless and $\log _{\sigma} 1$ is not defined therefore, in our study we take non-empty SVNSs and $\sigma \neq 1$, where $\sigma$ is any real number.

Definition 13. For any $S V N N \zeta_{p}=\left\langle P_{\xi_{p}}(r), I_{\xi_{p}}(r), N_{\xi_{p}}(r)\right\rangle$ in $\Re$. The logarithmic $S V N N$ is defined as:

$$
\log _{\sigma} \zeta_{p}=\left\{\left\langle 1-\left(\log _{\sigma} P_{\xi_{p}}(r)\right), \log _{\sigma}\left(1-I_{\tilde{\zeta}_{p}}(r)\right), \log _{\sigma}\left(1-N_{\tilde{\xi}_{p}}(r)\right)\right\rangle \mid r \in \Re\right\},
$$


in which $P_{\sigma}: \Re \rightarrow \Theta, I_{\sigma}: \Re \rightarrow \Theta$ and $N_{\sigma}: \Re \rightarrow \Theta$ are indicated the truth, indeterminacy and falsity memberships in $\Re, \Theta=[0,1]$ be the unit interval. Also following condition is satisfied by $P_{\sigma}, I_{\sigma}$ and $N_{\sigma}$, $0 \leq P_{\sigma}(r)+I_{\sigma}(r)+N_{\sigma}(r) \leq 3 ; \forall r \in \Re$. Therefore the truth membership grade is

$$
1-\left(\log _{\sigma} P_{\xi_{p}}(r)\right): \Re \rightarrow \Theta, \text { such that } 0 \leq 1-\left(\log _{\sigma} P_{\xi_{p}}(r)\right) \leq 1 \text {, for all } r \in \Re
$$

the indeterminacy membership is

$$
\log _{\sigma}\left(1-I_{\tilde{\zeta}_{p}}(r)\right): \Re \rightarrow \Theta, \text { such that } 0 \leq \log _{\sigma}\left(1-I_{\xi_{p}}(r)\right) \leq 1 \text {, for all } r \in \Re
$$

and falsity membership is

$$
\log _{\sigma}\left(1-N_{\xi_{p}}(r)\right): \Re \rightarrow \Theta, \text { such that } 0 \leq \log _{\sigma}\left(1-N_{\xi_{p}}(r)\right) \leq 1, \text { for all } r \in \Re
$$

Therefore

$$
\begin{aligned}
\log _{\sigma} \zeta_{p} & =\left\{\left\langle 1-\left(\log _{\sigma} P_{\xi_{p}}(r)\right), \log _{\sigma}\left(1-I_{\xi_{p}}(r)\right), \log _{\sigma}\left(1-N_{\xi_{p}}(r)\right)\right\rangle \mid r \in \Re\right\} \\
0 & <\sigma \leq \min \left\{P_{\xi_{p}}, 1-I_{\xi_{p}}, 1-N_{\xi_{p}}\right\} \leq 1, \sigma \neq 1
\end{aligned}
$$

is SVNS.

Definition 14. For any $S V N N \zeta_{p}=\left\langle P_{\xi_{p}}(r), I_{\xi_{p}}(r), N_{\xi_{p}}(r)\right\rangle$ in $\Re$. If

$$
\log _{\sigma} \zeta_{p}=\left\{\begin{array}{c}
\left(\begin{array}{c}
1-\left(\log _{\sigma} P_{\xi_{p}}(r)\right), \\
\log _{\sigma}\left(1-I_{\xi_{p}}(r)\right), \\
\log \left(1-N_{\xi_{p}}(r)\right)
\end{array}\right) \quad 0<\sigma \leq \min \left\{P_{\left.\xi_{p}, 1-I_{\xi_{p}}, 1-N_{\xi_{p}}\right\}<1}\left(\begin{array}{c}
1-\left(\log _{\frac{1}{\sigma}} P_{\xi_{p}}(r)\right), \\
\log _{\frac{1}{\sigma}}\left(1-I_{\xi_{p}}(r)\right), \\
\log _{\frac{1}{\sigma}}\left(1-N_{\xi_{p}}(r)\right)
\end{array}\right) \quad 0<\frac{1}{\sigma} \leq \min \left\{P_{\xi_{p}}, 1-I_{\xi_{p},}, 1-N_{\xi_{p}}\right\}<1,\right. \\
\sigma \neq 1
\end{array}\right.
$$

then the function $\log _{\sigma} \zeta_{p}$ is known to be a logarithmic operator for SVNS, and its value is said to be logarithmic $\operatorname{SVNN}(L-S V N N)$. Here, we take $\log _{\sigma} 0=0, \sigma>0, \sigma \neq 1$.

Theorem 1. [10] For any $S V N N \zeta_{p}=\left\langle P_{\xi_{p}}(r), I_{\xi_{p}}(r), N_{\xi_{p}}(r)\right\rangle$ in $\Re$, then $\log _{\sigma} \zeta_{p}$ is also be SVNN.

Now, we give some discussion on the basic properties of the L-SVNN.

Definition 15. For any two L-SVNNs $\log _{\sigma} \zeta_{p}=\left(\begin{array}{c}1-\left(\log _{\sigma} P_{\xi_{p}}(r)\right), \\ \log _{\sigma}\left(1-I_{\xi_{p}}(r)\right), \\ \log _{\sigma}\left(1-N_{\xi_{p}}(r)\right)\end{array}\right)$ and $\log _{\sigma} \zeta_{q}=$ $\left(\begin{array}{l}1-\left(\log _{\sigma} P_{\mathcal{\zeta}_{q}}(r)\right), \\ \log _{\sigma}\left(1-I_{\xi_{q}}(r)\right), \\ \log _{\sigma}\left(1-N_{\xi_{q}}(r)\right)\end{array}\right)$ in $\Re$ and $\beta \geq 0$.Then the logarithmic operations of L-SVNNs are propose as (1) $\log _{\sigma} \zeta_{p} \oplus \log _{\sigma} \zeta_{q}=\left\{\begin{array}{c}1-\left(\log _{\sigma} P_{\xi_{p}}(r)\right) \cdot\left(\log _{\sigma} P_{\xi_{q}}(r)\right), \\ \log _{\sigma}\left(1-I_{\xi_{p}}(r)\right) \cdot \log _{\sigma}\left(1-I_{\xi_{q}}(r)\right), \\ \log _{\sigma}\left(1-N_{\xi_{p}}(r)\right) \cdot \log _{\sigma}\left(1-N_{\xi_{q}}(r)\right)\end{array}\right\}$; 
(2) $\beta \cdot \log _{\sigma} \zeta_{p}=\left\{\begin{array}{c}1-\left(\log _{\sigma} P_{\xi_{p}}(r)\right)^{\beta}, \\ \left(\log _{\sigma}\left(1-I_{\xi_{p}}(r)\right)\right)^{\beta}, \\ \left(\log _{\sigma}\left(1-N_{\xi_{p}}(r)\right)\right)^{\beta}\end{array}\right\}$;

(3) $\log _{\sigma} \zeta_{p} \otimes \log _{\sigma} \zeta_{q}=\left\{\begin{array}{c}1-\left(\log _{\sigma} P_{\xi_{p}}(r)\right) \cdot 1-\left(\log _{\sigma} P_{\xi_{q}}(r)\right), \\ 1-\left(1-\log _{\sigma}\left(1-I_{\xi_{p}}(r)\right)\right) \cdot\left(1-\log _{\sigma}\left(1-I_{\xi_{q}}(r)\right)\right), \\ 1-\left(1-\log _{\sigma}\left(1-N_{\xi_{p}}(r)\right)\right) \cdot\left(1-\log _{\sigma}\left(1-N_{\xi_{q}}(r)\right)\right)\end{array}\right\}$;

(4) $\left(\log _{\sigma} \zeta_{p}\right)^{\beta}=\left\{\begin{array}{c}\left(1-\left(\log _{\sigma} P_{\xi_{p}}(r)\right)\right)^{\beta} \\ 1-\left(1-\log _{\sigma}\left(1-I_{\xi_{p}}(r)\right)\right)^{\beta} \\ 1-\left(1-\log _{\sigma}\left(1-N_{\xi_{p}}(r)\right)\right)^{\beta}\end{array}\right\}$.

Theorem 2. [10] For any two L-SVNNs $\log _{\sigma} \zeta_{p}=\left(\begin{array}{c}1-\left(\log _{\sigma} P_{\xi_{p}}(r)\right) \\ \log _{\sigma}\left(1-I_{\xi_{p}}(r)\right), \\ \log _{\sigma}\left(1-N_{\xi_{p}}(r)\right)\end{array}\right)(p=1,2)$ in $\Re$, with $0<$ $\sigma \leq \min \left\{P_{\xi_{p}}, 1-I_{\xi_{p}}, 1-N_{\xi_{p}}\right\}<1, \sigma \neq 1, \beta, \beta_{1}, \beta_{2}>0$ be any real numbers. Then

(1) $\beta\left(\log _{\sigma} \zeta_{1} \oplus \log _{\sigma} \zeta_{2}\right)=\beta \log _{\sigma} \zeta_{1} \oplus \beta \log _{\sigma} \zeta_{2}$;

(2) $\left(\log _{\sigma} \zeta_{1} \otimes \log _{\sigma} \zeta_{2}\right)^{\beta}=\left(\log _{\sigma} \zeta_{1}\right)^{\beta} \otimes\left(\log _{\sigma} \zeta_{2}\right)^{\beta}$;

(3) $\beta_{1} \log _{\sigma} \zeta_{1} \oplus \beta_{2} \log _{\sigma} \zeta_{1}=\left(\beta_{1}+\beta_{2}\right) \log _{\sigma} \zeta_{1}$;

(4) $\left(\log _{\sigma} \zeta_{1}\right)^{\beta_{1}} \otimes\left(\log _{\sigma} \zeta_{1}\right)^{\beta_{2}}=\left(\log _{\sigma} \zeta_{1}\right)^{\left(\beta_{1}+\beta_{2}\right)}$;

(5) $\left(\left(\log _{\sigma} \zeta_{1}\right)^{\beta_{1}}\right)^{\beta_{2}}=\left(\log _{\sigma} \zeta_{1}\right)^{\beta_{1} \beta_{2}}$.

Comparison Technique for L-SVNNs

Definition 16. [10] For any L-SVNN $\log _{\sigma} \zeta_{p}=\left(\begin{array}{c}1-\left(\log _{\sigma} P_{\xi_{p}}(r)\right) \text {, } \\ \log _{\sigma}\left(1-I_{\mathcal{\zeta}_{p}}(r)\right), \\ \log _{\sigma}\left(1-N_{\xi_{p}}(r)\right)\end{array}\right)$ in $\Re$. Then score and accuracy values are define as
(1) $\widetilde{S}\left(\log _{\sigma} \zeta_{p}\right)=1-\left(\log _{\sigma} P_{\xi_{p}}(r)\right)-\log _{\sigma}\left(1-I_{\xi_{p}}(r)\right)-\left(\log _{\sigma}\left(1-N_{\tilde{\xi}_{p}}(r)\right)\right)$
(2) $\widetilde{A}\left(\log _{\sigma} \zeta_{p}\right)=1-\left(\log _{\sigma} P_{\xi_{p}}(r)\right)+\log _{\sigma}\left(1-I_{\xi_{p}}(r)\right)+\left(\log _{\sigma}\left(1-N_{\xi_{p}}(r)\right)\right)$

The above defined score and accuracy values suggest which L-SVNN are greater than other L-SVNNs. The comparison technique is defined in the following definition.

Definition 17. For any L-SVNNs $\log _{\sigma} \zeta_{p}=\left(\begin{array}{c}1-\left(\log _{\sigma} P_{\xi_{p}}(r)\right), \\ \log _{\sigma}\left(1-I_{\xi_{p}}(r)\right), \\ \log _{\sigma}\left(1-N_{\xi_{p}}(r)\right)\end{array}\right)(p=1,2)$ in $\Re$. Then, comparison technique is proposed as:

(1) If $\widetilde{S}\left(\log _{\sigma} \zeta_{1}\right)<\widetilde{S}\left(\log _{\sigma} \zeta_{2}\right)$ then $\log _{\sigma} \zeta_{1}<\log _{\sigma} \zeta_{2}$,

(2) If $\widetilde{S}\left(\log _{\sigma} \zeta_{1}\right)>\widetilde{S}\left(\log _{\sigma} \zeta_{2}\right)$ then $\log _{\sigma} \zeta_{1}>\log _{\sigma} \zeta_{2}$,

(3) If $\widetilde{S}\left(\log _{\sigma} \zeta_{1}\right)=\widetilde{S}\left(\log _{\sigma} \zeta_{2}\right)$ then

(a) $\widetilde{A}\left(\log _{\sigma} \zeta_{1}\right)<\widetilde{A}\left(\log _{\sigma} \zeta_{2}\right)$ then $\log _{\sigma} \zeta_{1}<\log _{\sigma} \zeta_{2}$,

(b) $\widetilde{A}\left(\log _{\sigma} \zeta_{1}\right)>\widetilde{A}\left(\log _{\sigma} \zeta_{2}\right)$ then $\log _{\sigma} \zeta_{1}>\log _{\sigma} \zeta_{2}$,

(c) $\widetilde{A}\left(\log _{\sigma} \zeta_{1}\right)=\widetilde{A}\left(\log _{\sigma} \zeta_{2}\right)$ then $\log _{\sigma} \zeta_{1} \approx \log _{\sigma} \zeta_{2}$. 


\section{Logarithmic Aggregation Operators for L-SVNNs}

Now, we propose novel logarithmic hybrid aggregation operators for L-SVNNs based on logarithmic operations laws as follows:

\subsection{Logarithmic Hybrid Averaging Operator}

Definition 18. For any collection of $S V N N s \zeta_{p}=\left\langle P_{\xi_{p}}(r), I_{\xi_{p}}(r), N_{\xi_{p}}(r)\right\rangle(p=1, \ldots, n)$ in $\Re$, with $0<$ $\sigma_{p} \leq \min \left\{P_{\xi_{p}}, 1-I_{\xi_{p}}, 1-N_{\xi_{p}}\right\}<1, \sigma \neq 1$. The structure of logarithmic single valued neutrosophic hybrid weighted averaging (L-SVNHWA) operator is

$$
L-\operatorname{SVNHWA}\left(\zeta_{1}, \zeta_{2}, \ldots, \zeta_{n}\right)=\sum_{p=1}^{n} \omega_{p} \ell_{0} g_{\sigma_{p}} \zeta_{\eta(p)}^{*}
$$

where $\beta_{p}(p=1, \ldots, n)$ is the weighting vector with $\beta_{p} \geq 0$ and $\sum_{p=1}^{n} \beta_{p}=1$ and $p$ th biggest weighted value is $\zeta_{\eta(p)}^{*}\left(\zeta_{\eta(p)}^{*}=n \beta_{p} \zeta_{\eta(p)}, P \in N\right)$ consequently by total order $\zeta_{\eta(1)}^{*} \geq \zeta_{\eta(2)}^{*} \geq \ldots \geq \zeta_{\eta(n)}^{*}$. Also, the associated weights are $\omega=\left(\omega_{1}, \omega_{2}, \ldots, \omega_{n}\right)$ with $\omega_{p} \geq 0, \Sigma_{p=1}^{n} \omega_{p}=1$.

Theorem 3. For any collection of SVNNs $\zeta_{p}=\left\langle P_{\xi_{p}}(r), I_{\xi_{p}}(r), N_{\xi_{p}}(r)\right\rangle(p=1, \ldots, n)$ in $\Re$, with $0<$ $\sigma_{p} \leq \min \left\{P_{\xi_{p}}, 1-I_{\xi_{p}}, 1-N_{\xi_{p}}\right\}<1, \sigma \neq 1$. Then by using logarithmic operations and Definition 18, $L-S V N H W A$ is defined as

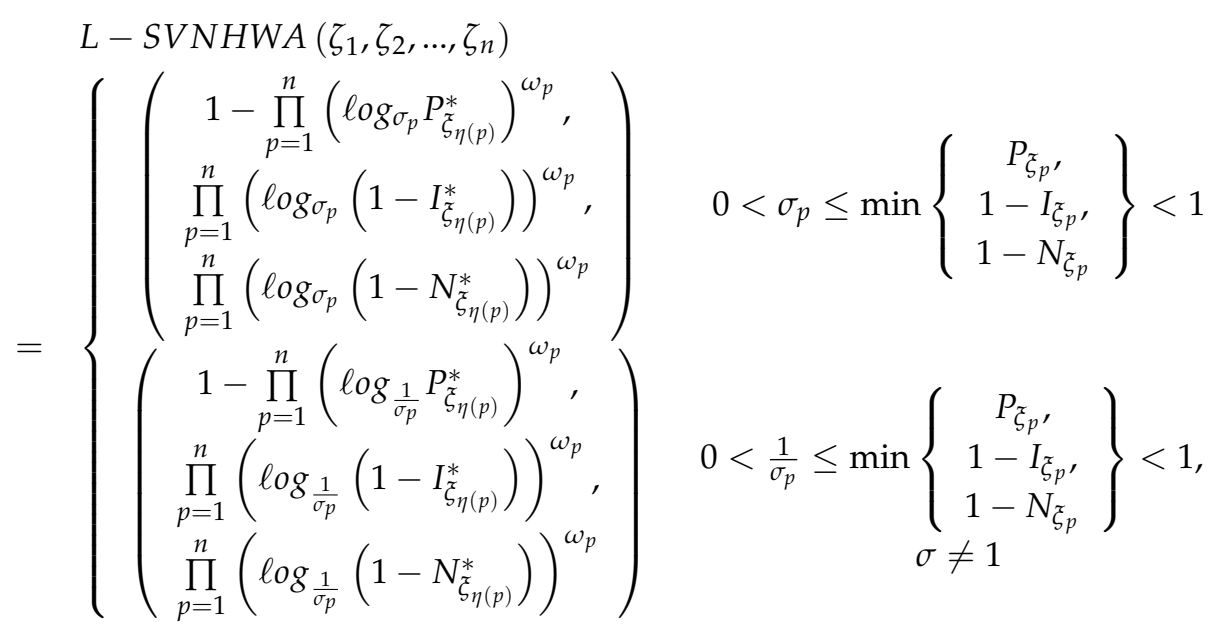

where $\beta_{p}(p=1, \ldots, n)$ are weighting vector with $\beta_{p} \geq 0$ and $\sum_{p=1}^{n} \beta_{p}=1$ and pth biggest weighted value is $\zeta_{\eta(p)}^{*}\left(\zeta_{\eta(p)}^{*}=n \beta_{p} \zeta_{\eta(p)}, P \in N\right)$ consequently by total order $\zeta_{\eta(1)}^{*} \geq \zeta_{\eta(2)}^{*} \geq \ldots \geq \zeta_{\eta(n)}^{*}$. Also the associated weights are $\omega=\left(\omega_{1}, \omega_{2}, \ldots, \omega_{n}\right)$ with $\omega_{p} \geq 0, \sum_{p=1}^{n} \omega_{p}=1$.

Proof. Using mathematical induction to prove Equation (3), we proceed as:

(a) For $n=2$, since

$$
\omega_{1} \log _{\sigma_{1}} \zeta_{\eta(1)}^{*}=\left(\begin{array}{c}
\left.1-\left(\log _{\sigma_{1}} P_{\xi_{\eta(1)}}^{*}\right)\right)^{\omega_{1}}, \\
\left(\log _{\sigma_{1}}\left(1-I_{\xi_{\eta(1)}}^{*}\right)\right)^{\omega_{1}}, \\
\left(\log _{\sigma_{1}}\left(1-N_{\xi_{\eta(1)}}^{*}\right)\right)^{\omega_{1}^{\prime}}
\end{array}\right)
$$


and

$$
\omega_{2} \log _{\sigma_{2}} \zeta_{\eta(2)}^{*}=\left(\begin{array}{c}
1-\left(\log _{\sigma_{2}} P_{\xi_{\eta(2)}^{*}}^{*}\right){ }^{\omega_{2}}, \\
\left(\log _{\sigma_{2}}\left(1-I_{\zeta_{\eta(2)}}^{*}\right)\right)^{\omega_{2}}, \\
\left(\log _{\sigma_{2}}\left(1-N_{\xi_{\eta(2)}}^{*}\right)\right)^{\omega_{2}}
\end{array}\right)
$$

Then

$$
\begin{aligned}
& L-\operatorname{SVNHWA}\left(\zeta_{1}, \zeta_{2}\right)=\omega_{1} \log _{\sigma_{1}} \zeta_{\eta(1)}^{*} \oplus \omega_{2} \log _{\sigma_{2}} \zeta_{\eta(2)}^{*} \\
& =\left(\begin{array}{c}
1-\left(\log _{\sigma_{1}} P_{\xi_{\eta(1)}^{*}}^{*}\right)^{\omega_{1}}, \\
\left(\log _{\sigma_{1}}\left(1-I_{\mathcal{\zeta}_{\eta(1)}}^{*}\right)\right)^{\omega_{1}}, \\
\left(\log _{\sigma_{1}}\left(1-N_{\xi_{\eta(1)}^{*}}^{*}\right)\right)^{\omega_{1}}
\end{array}\right) \oplus\left(\begin{array}{c}
1-\left(\log _{\sigma_{2}} P_{\xi_{\eta(2)}^{*}}^{*}\right)^{\omega_{2}}, \\
\left(\log _{\sigma_{2}}\left(1-I_{\xi_{\eta(2)}}^{*}\right)\right)^{\omega_{2}}, \\
\left(\log _{\sigma_{2}}\left(1-N_{\xi_{\eta(2)}}^{*}\right)\right)^{\omega_{2}}
\end{array}\right) \\
& =\left(\begin{array}{c}
1-\left(\log _{\sigma_{1}} P_{\xi_{\eta(1)}}^{*}\right)^{\omega_{1}} \cdot\left(\log _{\sigma_{2}} P_{\xi_{\eta(2)}}^{*}\right)^{\omega_{2}}, \\
\left(\log _{\sigma_{1}}\left(1-I_{\xi_{\eta(1)}^{*}}^{*}\right)\right)^{\omega_{1}} \cdot\left(\log _{\sigma_{2}}\left(1-I_{\xi_{\eta(2)}}^{*}\right)\right)^{\omega_{2}}, \\
\left(\log _{\sigma_{1}}\left(1-N_{\xi_{\eta(1)}}^{*}\right)\right)^{\omega_{1}} \cdot\left(\log _{\sigma_{2}}\left(1-N_{\xi_{\eta(2)}}^{*}\right)\right)^{\omega_{2}}
\end{array}\right) \\
& =\left(\begin{array}{c}
1-\prod_{p=1}^{2}\left(\log _{\sigma_{p}} P_{\xi_{\eta(p)}^{*}}^{*}\right)^{\omega_{p}}, \\
\prod_{p=1}^{n}\left(\log _{\sigma_{p}}\left(1-I_{\xi_{\eta(p)}^{*}}^{*}\right)\right)^{\omega_{p}}, \\
\prod_{p=1}^{n}\left(\log _{\sigma_{p}}\left(1-N_{\xi_{\eta(p)}}^{*}\right)\right)^{\omega_{p}}
\end{array}\right) .
\end{aligned}
$$

(b) Now Equation (3) is true for $n=k$,

$$
L-\operatorname{SVNHWA}\left(\zeta_{1}, \zeta_{2}, \ldots, \zeta_{k}\right)=\left(\begin{array}{c}
1-\prod_{p=1}^{k}\left(\log _{\sigma_{p}} P_{\xi_{\eta(p)}}^{*}\right)^{\omega_{p}}, \\
\prod_{p=1}^{k}\left(\log _{\sigma_{p}}\left(1-I_{\xi_{\eta(p)}}^{*}\right)\right)^{\omega_{p}}, \\
\prod_{p=1}^{k}\left(\log _{\sigma_{p}}\left(1-N_{\xi_{\eta(p)}}^{*}\right)\right)^{\omega_{p}}
\end{array}\right) .
$$

(c) Now, we prove that Equation (3) for $n=k+1$, that is

$$
L-\operatorname{SVNHWA}\left(\zeta_{1}, \zeta_{2}, \ldots, \zeta_{k}\right)=\sum_{p=1}^{k} \omega_{p} \log _{\sigma_{p}} \zeta_{\eta(p)}^{*}+\omega_{k+1} \log _{\sigma_{k+1}} \zeta_{\eta(k+1)}^{*}
$$




$$
\begin{aligned}
& L-\operatorname{SVNHWA}\left(\zeta_{1}, \zeta_{2}, \ldots, \zeta_{k}\right) \\
= & \left(\begin{array}{c}
1-\prod_{p=1}^{k}\left(\log _{\sigma_{p}} P_{\xi_{\eta(p)}^{*}}^{*}\right)^{\omega_{p}}, \\
\prod_{p=1}^{k}\left(\log _{\sigma_{p}}\left(1-I_{\xi_{\eta(p)}}^{*}\right)\right)^{\omega_{p}}, \\
\prod_{p=1}^{k}\left(\log _{\sigma_{p}}\left(1-N_{\xi_{\eta(p)}}^{*}\right)\right)^{\omega_{p}}
\end{array}\right) \oplus\left(\begin{array}{c}
1-\left(\log _{\sigma_{k+1}} P_{\xi_{\eta(k+1)}}^{*}\right)^{\omega_{k+1}}, \\
\left(\log _{\sigma_{k+1}}\left(1-I_{\xi_{\eta(k+1)}}^{*}\right)\right)^{\omega_{k+1}}, \\
\left(\log _{\sigma_{k+1}}\left(1-N_{\xi_{\eta(k+1)}}^{*}\right)\right)^{\omega_{k+1}}
\end{array}\right) \\
= & \left(\begin{array}{c}
1-\prod_{p=1}^{k+1}\left(\log _{\sigma_{p}} P_{\xi_{\eta(p)}^{*}}^{*}\right)^{\omega_{p}}, \\
\prod_{p=1}^{k+1}\left(\log _{\sigma_{p}}\left(1-I_{\xi_{\eta(p)}^{*}}^{*}\right)\right)^{\omega_{p}}, \\
\prod_{p=1}\left(\log _{\sigma_{p}}\left(1-N_{\xi_{\eta(p)}}^{*}\right)\right)^{\omega_{p}}
\end{array}\right)
\end{aligned}
$$

Thus Equation (3) is true for $n=z+1$. Hence its satisfies for whole $n$. Therefore

$$
L-\operatorname{SVNHWA}\left(\zeta_{1}, \zeta_{2}, \ldots, \zeta_{n}\right)=\left(\begin{array}{c}
1-\prod_{p=1}^{n}\left(\log _{\sigma_{p}} P_{\xi_{\eta(p)}}^{*}\right)^{\omega_{p}}, \\
\prod_{p=1}^{n}\left(\log _{\sigma_{p}}\left(1-I_{\xi_{\eta(p)}}^{*}\right)\right)^{\omega_{p}}, \\
\prod_{p=1}^{n}\left(\log _{\sigma_{p}}\left(1-N_{\xi_{\eta(p)}}^{*}\right)\right)^{\omega_{p}}
\end{array}\right)
$$

In a similarly way, if $0<\frac{1}{\sigma_{p}} \leq \min \left\{P_{\xi_{p}}, 1-I_{\xi_{p}}, 1-N_{\xi_{p}}\right\}<1, \sigma \neq 1$, we can also obtain

$$
L-\operatorname{SVNHWA}\left(\zeta_{1}, \zeta_{2}, \ldots, \zeta_{n}\right)=\left(\begin{array}{c}
1-\prod_{p=1}^{n}\left(\log _{\frac{1}{\sigma_{p}}} P_{\xi_{\eta(p)}}^{*}\right)^{\omega_{p}}, \\
\prod_{p=1}^{n}\left(\log _{\frac{1}{\sigma_{p}}}\left(1-I_{\xi_{\eta(p)}}^{*}\right)\right)^{\omega_{p}}, \\
\prod_{p=1}^{n}\left(\log _{\frac{1}{\sigma_{p}}}\left(1-N_{\xi_{\eta(p)}}^{*}\right)\right)^{\omega_{p}}
\end{array}\right)
$$

which completes the proof.

Remark 1. If $\sigma_{1}=\sigma_{2}=\sigma_{3}=\ldots=\sigma_{n}=\sigma$, that is $0<\sigma \leq \min \left\{P_{\xi_{p}}, 1-I_{\xi_{p}}, 1-N_{\xi_{p}}\right\}<1, \sigma \neq 1$, then $L-S V N H W A$ operator is reduced as follows

$$
L-\operatorname{SVNHWA}\left(\zeta_{1}, \zeta_{2}, \ldots, \zeta_{n}\right)=\left(\begin{array}{c}
1-\prod_{p=1}^{n}\left(\log _{\sigma} P_{\xi_{\eta(p)}}^{*}\right)^{\omega_{p}}, \\
\prod_{p=1}^{n}\left(\log _{\sigma}\left(1-I_{\xi_{\eta(p)}}^{*}\right)\right)^{\omega_{p}}, \\
\prod_{p=1}^{n}\left(\log _{\sigma}\left(1-N_{\xi_{\eta(p)}}^{*}\right)\right)^{\omega_{p}}
\end{array}\right) .
$$

\section{Properties}

$L-S V N H W A$ operator satisfies some properties are enlist below;

(1) Idempotency: For any collection of SVNNs $\zeta_{p}=\left\langle P_{\xi_{p}}(r), I_{\xi_{p}}(r), N_{\xi_{p}}(r)\right\rangle(p=1, \ldots, n)$ in $\Re$. Then, if collection of SVNNs $\zeta_{p}=\left\langle P_{\xi_{p}}(r), I_{\xi_{p}}(r), N_{\xi_{p}}(r)\right\rangle(p=1, \ldots, n)$ are identical, that is

$$
L-\operatorname{SVNHWA}\left(\zeta_{1}, \zeta_{2}, \ldots, \zeta_{n}\right)=\zeta \text {. }
$$


(2) Boundedness: for any collection of SVNNs $\zeta_{p}=\left\langle P_{\xi_{p}}(r), I_{\xi_{p}}(r), N_{\xi_{p}}(r)\right\rangle(p=1, \ldots, n)$ in $\Re . \zeta_{p}^{-}=\left\langle\min _{p} P_{\xi_{p}}^{*}, \max _{p} I_{\xi_{p}}^{*}, \max _{p} N_{\xi_{p}}^{*}\right\rangle$ and $\zeta_{p}^{+}=\left\langle\max _{p} P_{\xi^{\prime} p^{\prime}}^{*} \min _{p} I_{\xi^{\prime}}^{*}, \min _{p} N_{\xi_{p}}^{*}\right\rangle(p=1, \ldots, n)$ in $\Re$, therefore

$$
\zeta_{p}^{-} \subseteq L-S V N H W A\left(\zeta_{1}, \zeta_{2}, \ldots, \zeta_{n}\right) \subseteq \zeta_{p}^{+} .
$$

(3) Monotonically: for any collection of SVNNs $\zeta_{p}=\left\langle P_{\xi_{p}}(r), I_{\xi_{p}}(r), N_{\xi_{p}}(r)\right\rangle(p=1, \ldots, n)$ in $\Re$. If $\zeta_{\eta(p)} \subseteq \zeta_{\eta(p)}^{*}$ for $(p=1, \ldots, n)$, then

$$
L-\operatorname{SVNHWA}\left(\zeta_{1}, \zeta_{2}, \ldots, \zeta_{n}\right) \subseteq L-\operatorname{SVNHWA}\left(\zeta_{1}^{*}, \zeta_{2}^{*}, \ldots, \zeta_{n}^{*}\right)
$$

\subsection{Logarithmic Hybrid Geometric Operators}

Definition 19. For any collection of SVNNs $\zeta_{p}=\left\langle P_{\xi_{p}}(r), I_{\xi_{p}}(r), N_{\xi_{p}}(r)\right\rangle(p=1, \ldots, n)$ in $\Re$, with $0<$ $\sigma_{p} \leq \min \left\{P_{\xi_{p}}, 1-I_{\xi_{p}}, 1-N_{\xi_{p}}\right\}<1, \sigma \neq 1$. The structure of logarithmic single valued neutrosophic hybrid weighted geometric (L-SVNHWG) operator is

$$
L-\operatorname{SVNHWG}\left(\zeta_{1}, \zeta_{2}, \ldots, \zeta_{n}\right)=\prod_{p=1}^{n}\left(\log _{\sigma_{p}} \zeta_{\eta(p)}^{*}\right)^{\omega_{p}}
$$

where $\beta_{p}(p=1, \ldots, n)$ are weight vectors with $\beta_{p} \geq 0$ and $\sum_{p=1}^{n} \beta_{p}=1$ and $p$ th biggest weighted value is $\zeta_{\eta(p)}^{*}\left(\zeta_{\eta(p)}^{*}=\left(\zeta_{\eta(p)}\right)^{n \beta_{p}}, P \in N\right)$ consequently by total order $\zeta_{\eta(1)}^{*} \geq \zeta_{\eta(2)}^{*} \geq \ldots \geq \zeta_{\eta(n)}^{*}$. Also associated weights are $\omega=\left(\omega_{1}, \omega_{2}, \ldots, \omega_{n}\right)$ with $\omega_{p} \geq 0, \sum_{p=1}^{n} \omega_{p}=1$.

Theorem 4. For any collection of SVNNs $\zeta_{p}=\left\langle P_{\mathcal{\zeta}_{p}}(r), I_{\tilde{\xi}_{p}}(r), N_{\tilde{\xi}_{p}}(r)\right\rangle(p=1, \ldots, n)$ in $\Re$, with $0<$ $\sigma_{p} \leq \min \left\{P_{\xi_{p}}, 1-I_{\xi_{p}}, 1-N_{\xi_{p}}\right\}<1, \sigma \neq 1$. Then by using logarithmic operations and Definition 19, $L-S V N H W G$ define as

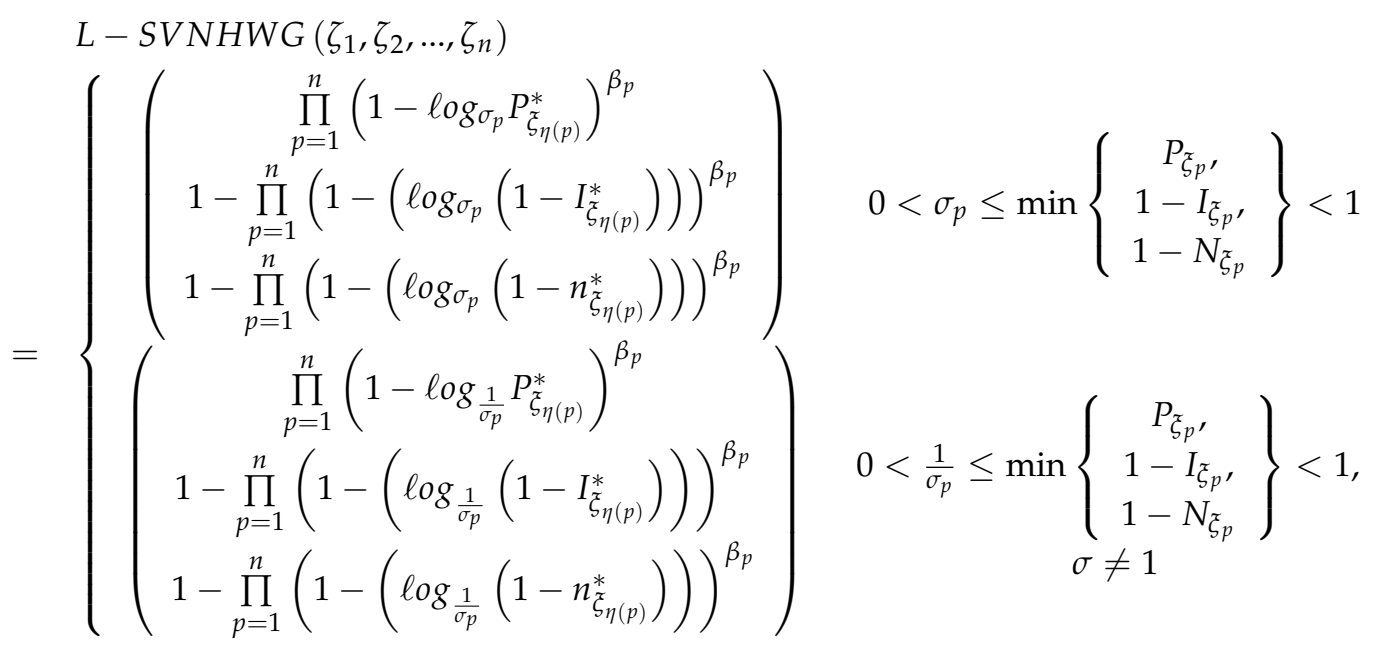

where $\beta_{p}(p=1, \ldots, n)$ are weight vectors with $\beta_{p} \geq 0$ and $\sum_{p=1}^{n} \beta_{p}=1$ and $p$ th biggest weighted value is $\zeta_{\eta(p)}^{*}\left(\zeta_{\eta(p)}^{*}=\left(\zeta_{\eta(p)}\right)^{n \beta_{p}}, P \in N\right)$ consequently by total order $\zeta_{\eta(1)}^{*} \geq \zeta_{\eta(2)}^{*} \geq \ldots \geq \zeta_{\eta(n)}^{*}$. Also associated weights are $\omega=\left(\omega_{1}, \omega_{2}, \ldots, \omega_{n}\right)$ with $\omega_{p} \geq 0, \sum_{p=1}^{n} \omega_{p}=1$.

Proof. Using mathematical induction to prove Equation (4), we proceed as: 
(a) For $n=2$, since

$$
\left(\log _{\sigma_{1}} \zeta_{1}^{*}\right)^{\omega_{1}}=\left(\begin{array}{c}
\left(1-\log _{\sigma_{1}} P_{\xi_{1}}^{*}\right)^{\omega_{1}} \\
1-\left(1-\left(\log _{\sigma_{1}}\left(1-I_{\xi_{1}}^{*}\right)\right)\right)^{\omega_{1}} \\
1-\left(1-\left(\log _{\sigma_{1}}\left(1-N_{\xi_{1}}^{*}\right)\right)\right)^{\omega_{1}}
\end{array}\right)
$$

and

$$
\left(\log _{\sigma_{2}} \zeta_{2}^{*}\right)^{\omega_{2}}=\left(\begin{array}{c}
\left(1-\log _{\sigma_{2}} P_{\xi_{2}}^{*}\right)^{\omega_{2}} \\
1-\left(1-\left(\log _{\sigma_{2}}\left(1-I_{\xi_{2}}^{*}\right)\right)\right)^{\omega_{2}} \\
1-\left(1-\left(\log _{\sigma_{2}}\left(1-N_{\xi_{2}}^{*}\right)\right)\right)^{\omega_{2}}
\end{array}\right)
$$

Then

$$
\begin{aligned}
& L-\operatorname{SVNHWG}\left(\zeta_{1}, \zeta_{2}\right)=\left(\log _{\sigma_{1}} \zeta_{1}^{*}\right)^{\omega_{1}} \otimes\left(\log _{\sigma_{2}} \zeta_{2}^{*}\right)^{\omega_{2}} \\
& =\left(\begin{array}{c}
\left(1-\log _{\sigma_{1}} P_{\xi_{1}}^{*}\right)^{\omega_{1}} \\
1-\left(1-\left(\log _{\sigma_{1}}\left(1-I_{\xi_{1}}^{*}\right)\right)\right)^{\omega_{1}} \\
1-\left(1-\left(\log _{\sigma_{1}}\left(1-N_{\xi_{1}}^{*}\right)\right)\right)^{\omega_{1}}
\end{array}\right) \otimes\left(\begin{array}{c}
\left(1-\log _{\sigma_{2}} P_{\xi_{2}}^{*}\right)^{\omega_{2}} \\
1-\left(1-\left(\log _{\sigma_{2}}\left(1-I_{\mathcal{\zeta}_{2}}^{*}\right)\right)\right)^{\omega_{2}} \\
1-\left(1-\left(\log _{\sigma_{2}}\left(1-N_{\xi_{2}}^{*}\right)\right)\right)^{\omega_{2}}
\end{array}\right) \\
& =\left\{\begin{array}{c}
\left(1-\log _{\sigma_{1}} P_{\xi_{1}}^{*}\right)^{\omega_{1}} \cdot\left(1-\log _{\sigma_{2}} P_{\xi_{2}}^{*}\right)^{\omega_{2}} \\
1-\left(1-\left(\log _{\sigma_{1}}\left(1-I_{\xi_{1}}^{*}\right)\right)\right)^{\omega_{1}} \cdot\left(1-\left(\log _{\sigma_{2}}\left(1-I_{\xi_{2}}^{*}\right)\right)\right)^{\omega_{2}} \\
1-\left(1-\left(\log _{\sigma_{1}}\left(1-N_{\xi_{1}}^{*}\right)\right)\right)^{\omega_{1}} \cdot\left(1-\left(\log _{\sigma_{2}}\left(1-N_{\xi_{2}}^{*}\right)\right)\right)^{\omega_{2}}
\end{array}\right\} \\
& =\left(\begin{array}{c}
\prod_{p=1}^{2}\left(1-\log _{\sigma_{p}} P_{\xi_{p}}^{*}\right)^{\omega_{p}}, \\
1-\prod_{p=1}^{2}\left(1-\left(\log _{\sigma_{p}}\left(1-I_{\xi_{p}}^{*}\right)\right)\right)^{\omega_{p}} \\
1-\prod_{p=1}^{2}\left(1-\left(\log _{\sigma_{p}}\left(1-N_{\xi_{p}}^{*}\right)\right)\right)^{\omega_{p}}
\end{array}\right) .
\end{aligned}
$$

(b) Now Equation (4) is true for $n=k$,

$$
L-\operatorname{SVNHWG}\left(\zeta_{1}, \zeta_{2}, \ldots, \zeta_{k}\right)=\left(\begin{array}{c}
\prod_{p=1}^{k}\left(1-\log _{\sigma_{p}} P_{\xi_{p}}^{*}\right)^{\omega_{p}} \\
1-\prod_{p=1}^{k}\left(1-\left(\log _{\sigma_{p}}\left(1-I_{\xi_{p}}^{*}\right)\right)\right)^{\omega_{p}} \\
1-\prod_{p=1}^{k}\left(1-\left(\log _{\sigma_{p}}\left(1-N_{\zeta_{p}}^{*}\right)\right)\right)^{\omega_{p}}
\end{array}\right),
$$

(c) Now, we prove that Equation (4) for $n=k+1$, that is

$$
L-\operatorname{SVNHWG}\left(\zeta_{1}, \zeta_{2}, \ldots, \zeta_{k}, \zeta_{k+1}\right)=\prod_{p=1}^{k}\left(\log _{\sigma_{p}} \zeta_{p}\right)^{\omega_{p}} \otimes\left(\log _{\sigma_{k+1}} \zeta_{k+1}\right)^{\omega_{k+1}}
$$




$$
\begin{aligned}
& L-\operatorname{SVNHWG}\left(\zeta_{1}, \zeta_{2}, \ldots, \zeta_{k}, \zeta_{k+1}\right) \\
= & \left(\begin{array}{c}
\prod_{p=1}^{k}\left(1-\log _{\sigma_{p}} P_{\xi_{p}}^{*}\right)^{\omega_{p}} \\
1-\prod_{p=1}^{k}\left(1-\left(\log _{\sigma_{p}}\left(1-I_{\xi_{p}}^{*}\right)\right)\right)^{\omega_{p}} \\
1-\prod_{p=1}^{k}\left(1-\left(\log _{\sigma_{p}}\left(1-N_{\xi_{p}}^{*}\right)\right)\right)^{\omega_{p}}
\end{array}\right) \otimes\left(\begin{array}{c}
\left(1-\log _{\sigma_{p}} P_{\xi_{k+1}}^{*}\right)^{\omega_{k+1}} \\
1-\left(1-\left(\log _{\sigma_{p}}\left(1-I_{\xi_{k+1}}^{*}\right)\right)\right)^{\omega_{k+1}} \\
1-\left(1-\left(\log _{\sigma_{p}}\left(1-N_{\xi_{k+1}}^{*}\right)\right)\right)^{\omega_{k+1}}
\end{array}\right) \\
= & \left(\begin{array}{c}
\prod_{p=1}^{k+1}\left(1-\log _{\sigma_{p}} P_{\xi_{p}}^{*}\right)^{\omega_{p}} \\
1-\prod_{p=1}^{k+1}\left(1-\left(\log _{\sigma_{p}}\left(1-I_{\xi_{p}}^{*}\right)\right)\right)^{\omega_{p}} \\
1-\prod_{p=1}^{k+1}\left(1-\left(\log _{\sigma_{p}}\left(1-N_{\xi_{p}}^{*}\right)\right)\right)^{\omega_{p}}
\end{array}\right)
\end{aligned}
$$

Thus Equation (4) is true for $n=z+1$. Hence it is satisfied for all $n$. Therefore

$$
L-\operatorname{SVNHWG}\left(\zeta_{1}, \zeta_{2}, \ldots, \zeta_{n}\right)=\left(\begin{array}{c}
\prod_{p=1}^{n}\left(1-\log _{\sigma_{p}} P_{\xi_{p}}^{*}\right)^{\omega_{p}} \\
1-\prod_{p=1}^{n}\left(1-\left(\log _{\sigma_{p}}\left(1-I_{\xi_{p}}^{*}\right)\right)\right)^{\omega_{p}} \\
1-\prod_{p=1}^{n}\left(1-\left(\log _{\sigma_{p}}\left(1-N_{\xi_{p}}^{*}\right)\right)\right)^{\omega_{p}}
\end{array}\right) .
$$

In a similar way, if $0<\frac{1}{\sigma_{p}} \leq \min \left\{P_{\xi_{p}}, 1-I_{\xi_{p}}, 1-N_{\xi_{p}}\right\}<1, \sigma \neq 1$, we can also obtain

$$
L-\operatorname{SVNHWG}\left(\zeta_{1}, \zeta_{2}, \ldots, \zeta_{n}\right)=\left(\begin{array}{c}
\prod_{p=1}^{n}\left(1-\log _{\frac{1}{\sigma_{p}}} P_{\xi_{p}}^{*}\right)^{\omega_{p}} \\
1-\prod_{p=1}^{n}\left(1-\left(\log _{\frac{1}{\sigma_{p}}}\left(1-I_{\xi_{p}}^{*}\right)\right)\right)^{\omega_{p}} \\
1-\prod_{p=1}^{n}\left(1-\left(\log _{\frac{1}{\sigma_{p}}}\left(1-N_{\xi_{p}}^{*}\right)\right)\right)^{\omega_{p}}
\end{array}\right)
$$

which completes the proof.

Remark 2. If $\sigma_{1}=\sigma_{2}=\sigma_{3}=\ldots=\sigma_{n}=\sigma$, that is $0<\sigma \leq \min \left\{P_{\xi_{p}}, 1-I_{\xi_{p}}, 1-N_{\xi_{p}}\right\}<1, \sigma \neq 1$, then $L-S V N H W G$ operator reduced as follows

$$
L-\operatorname{SVNHWG}\left(\zeta_{1}, \zeta_{2}, \ldots, \zeta_{n}\right)=\left(\begin{array}{c}
\prod_{p=1}^{n}\left(1-\log _{\sigma} P_{\xi_{p}}^{*}\right)^{\omega_{p}} \\
1-\prod_{p=1}^{n}\left(1-\left(\log _{\sigma}\left(1-I_{\xi_{p}}^{*}\right)\right)\right)^{\omega_{p}} \\
1-\prod_{p=1}^{n}\left(1-\left(\log _{\sigma}\left(1-N_{\xi_{p}}^{*}\right)\right)\right)^{\omega_{p}}
\end{array}\right) .
$$

\section{Properties}

$L-S V N H W G$ operator satisfies some properties are enlist below;

(1) Idempotency: for any collection of SVNNs $\zeta_{p}=\left\langle P_{\xi_{p}}(r), I_{\xi_{p}}(r), N_{\xi_{p}}(r)\right\rangle(p=1, \ldots, n)$ in $\Re$. Then, if collection of SVNNs $\zeta_{p}=\left\langle P_{\xi_{p}}(r), I_{\xi_{p}}(r), N_{\xi_{p}}(r)\right\rangle(p=1, \ldots, n)$ are identical, that is

$$
L-\operatorname{SVNHWG}\left(\zeta_{1}, \zeta_{2}, \ldots, \zeta_{n}\right)=\zeta \text {. }
$$


(2) Boundedness: for any collection of SVNNs $\zeta_{p}=\left\langle P_{\xi_{p}}(r), I_{\xi_{p}}(r), N_{\xi_{p}}(r)\right\rangle(p=1, \ldots, n)$ in $\Re . \zeta_{p}^{-}=\left\langle\min _{p} P_{\xi_{p}}, \max _{p} I_{\xi_{p}}, \max _{p} N_{\xi_{p}}\right\rangle$ and $\zeta_{p}^{+}=\left\langle\max _{p} P_{\xi_{p}}, \min _{p} I_{\xi_{p}}, \min _{p} N_{\xi_{p}}\right\rangle(p=1, \ldots, n)$ in $\Re$, therefore

$$
\zeta_{p}^{-} \subseteq L-S V N H W G\left(\zeta_{1}, \zeta_{2}, \ldots, \zeta_{n}\right) \subseteq \zeta_{p}^{+} .
$$

(3) Monotonically: for any collection of SVNNs $\zeta_{p}=\left\langle P_{\xi_{p}}(r), I_{\xi_{p}}(r), N_{\xi_{p}}(r)\right\rangle(p=1, \ldots, n)$ in $\Re$. If $\zeta_{p} \subseteq \zeta_{p}^{*}$ for $(p=1, \ldots, n)$, then

$$
L-\operatorname{SVNHWG}\left(\zeta_{1}, \zeta_{2}, \ldots, \zeta_{n}\right) \subseteq L-\operatorname{SVNHWG}\left(\zeta_{1}^{*}, \zeta_{2}^{*}, \ldots, \zeta_{n}^{*}\right)
$$

\subsection{Generalized Logarithmic Averaging Operator}

Definition 20. For any collection of $S V N N s \zeta_{p}=\left\langle P_{\xi_{p}}(r), I_{\xi_{p}}(r), N_{\xi_{p}}(r)\right\rangle(p=1, \ldots, n)$ in $\Re$, with $0<$ $\sigma_{p} \leq \min \left\{P_{\xi_{p}}, 1-I_{\xi_{p}}, 1-N_{\xi_{p}}\right\}<1, \sigma \neq 1$. The structure of logarithmic generalized single-valued neutrosophic weighted averaging (L-GSVNWA) operator is

$$
L-\operatorname{GSVNWA}\left(\zeta_{1}, \zeta_{2}, \ldots, \zeta_{n}\right)=\left(\sum_{p=1}^{n} \beta_{p} \log _{\sigma_{p}}\left(\zeta_{p}\right)^{\gamma}\right)^{\frac{1}{\gamma}}
$$

where $\beta_{p}(p=1, \ldots, n)$ are weighting vector with $\beta_{p} \geq 0$ and $\sum_{p=1}^{n} \beta_{p}=1$.

Theorem 5. For any collection of SVNNs $\zeta_{p}=\left\langle P_{\xi_{p}}(r), I_{\tilde{\xi}_{p}}(r), N_{\tilde{\xi}_{p}}(r)\right\rangle(p=1, \ldots, n)$ in $\Re$, with $0<$ $\sigma_{p} \leq \min \left\{P_{\xi_{p}}, 1-I_{\xi_{p}}, 1-N_{\xi_{p}}\right\}<1, \sigma \neq 1, \gamma \geq 1$. Then by using logarithmic operations and Definition 20, $L-G S V N W A$ define as

$$
\begin{aligned}
& L-\operatorname{GSVNWA}\left(\zeta_{1}, \zeta_{2}, \ldots, \zeta_{n}\right) \\
& \left\{\begin{array}{c}
\left(1-\prod_{p=1}^{n}\left(1-\left(1-\left(\log _{\sigma_{p}} P_{\xi_{p}}\right)\right)^{\gamma}\right)^{\beta_{p}}\right)^{\frac{1}{\gamma}}, \\
1-\left[1-\prod_{p=1}^{n}\left(1-\left(1-\log _{\sigma_{p}}\left(1-I_{\xi_{p}}\right)\right)^{\gamma}\right)^{\beta_{p}}\right]^{\frac{1}{\gamma}} \\
1-\left[1-\prod_{p=1}^{n}\left(1-\left(1-\log _{\sigma_{p}}\left(1-N_{\xi_{p}}\right)\right)^{\gamma}\right)^{\beta_{p}}\right]^{\frac{1}{\gamma}}
\end{array}\right) \quad 0<\sigma_{p} \leq \min \left\{\begin{array}{c}
P_{\xi_{p}} \\
1-I_{\xi_{p}}, \\
1-N_{\xi_{p}}
\end{array}\right\}<1 \\
& \left.\left(\begin{array}{c}
\left(1-\prod_{p=1}^{n}\left(1-\left(1-\left(\log _{\frac{1}{\sigma_{p}}} P_{\xi_{p}}\right)\right)^{\gamma}\right)^{\beta_{p}}\right)^{\frac{1}{\gamma}}, \\
1-\left[1-\prod_{p=1}^{n}\left(1-\left(1-\log _{\frac{1}{\sigma_{p}}}\left(1-I_{\xi_{p}}\right)\right)^{\gamma}\right)^{\beta_{p}}\right]^{\frac{1}{\gamma}} \\
1-\left[1-\prod_{p=1}^{n}\left(1-\left(1-\log _{\frac{1}{\sigma_{p}}}\left(1-N_{\xi_{p}}\right)\right)^{\gamma}\right)^{\beta_{p}}\right]^{\frac{1}{\gamma}}
\end{array}\right) \quad \begin{array}{c}
P_{\xi_{p},} \\
1-I_{\xi_{p}} \\
1-N_{\xi_{p}}
\end{array}\right\}<1,
\end{aligned}
$$

where $\beta_{p}(p=1, \ldots, n)$ are weighting vector with $\beta_{p} \geq 0$ and $\sum_{p=1}^{n} \beta_{p}=1$.

Apparently, if we use $\gamma=1$, then the $L-G S V N W A$ operator is becomes into $L-S V N W A$ operator.

Proof. Theorem 5 take the form by utilized the technique of mathematical induction and procedure is eliminate here. 
Remark 3. If $\sigma_{1}=\sigma_{2}=\sigma_{3}=\ldots=\sigma_{n}=\sigma$, that is $0<\sigma \leq \min \left\{P_{\xi_{p}}, 1-I_{\xi_{p}}, 1-N_{\xi_{p}}\right\}<1, \sigma \neq 1$, then $L-G S V N W A$ operator reduced as follows

$$
L-\operatorname{GSVNWA}\left(\zeta_{1}, \zeta_{2}, \ldots, \zeta_{n}\right)=\left(\begin{array}{c}
\left(1-\prod_{p=1}^{n}\left(1-\left(1-\left(\log _{\sigma} P_{\xi_{p}}\right)\right)^{\gamma}\right)^{\beta_{p}}\right)^{\frac{1}{\gamma}}, \\
1-\left[1-\prod_{p=1}^{n}\left(1-\left(1-\log _{\sigma}\left(1-I_{\xi_{p}}\right)\right)^{\gamma}\right)^{\beta_{p}}\right]^{\frac{1}{\gamma}} \\
1-\left[1-\prod_{p=1}^{n}\left(1-\left(1-\log _{\sigma}\left(1-N_{\xi_{p}}\right)\right)^{\gamma}\right)^{\beta_{p}}\right]^{\frac{1}{\gamma}}
\end{array}\right) .
$$

\section{Properties}

L - GSVNWA operator satisfies some properties are enlist below;

(1) Idempotency: For any collection of SVNNs $\zeta_{p}=\left\langle P_{\xi_{p}}(r), I_{\xi_{p}}(r), N_{\xi_{p}}(r)\right\rangle(p=1, \ldots, n)$ in $\Re$. Then, if collection of SVNNs $\zeta_{p}=\left\langle P_{\xi_{p}}(r), I_{\xi_{p}}(r), N_{\xi_{p}}(r)\right\rangle(p=1, \ldots, n)$ are identical, that is

$$
L-\operatorname{GSVNWA}\left(\zeta_{1}, \zeta_{2}, \ldots, \zeta_{n}\right)=\zeta
$$

(2) Boundedness: for any collection of SVNNs $\zeta_{p}=\left\langle P_{\xi_{p}}(r), I_{\xi_{p}}(r), N_{\xi_{p}}(r)\right\rangle(p=1, \ldots, n)$ in $\Re . \zeta_{p}^{-}=\left\langle\min _{p} P_{\xi_{p}}, \max _{p} I_{\xi_{p}}, \max _{p} N_{\xi_{p}}\right\rangle$ and $\zeta_{p}^{+}=\left\langle\max _{p} P_{\xi_{p}}, \min _{p} I_{\xi_{p}}, \min _{p} N_{\xi_{p}}\right\rangle(p=1, \ldots, n)$ in $\Re$, therefore

$$
\zeta_{p}^{-} \subseteq L-G S V N W A\left(\zeta_{1}, \zeta_{2}, \ldots, \zeta_{n}\right) \subseteq \zeta_{p}^{+} .
$$

(3) Monotonically: for any collection of SVNNs $\zeta_{p}=\left\langle P_{\xi_{p}}(r), I_{\xi_{p}}(r), N_{\xi_{p}}(r)\right\rangle(p=1, \ldots, n)$ in $\Re$. If $\zeta_{p} \subseteq \zeta_{p}^{*}$ for $(p=1, \ldots, n)$, then

$$
L-\operatorname{GSVNWA}\left(\zeta_{1}, \zeta_{2}, \ldots, \zeta_{n}\right) \subseteq L-\operatorname{GSVNWA}\left(\zeta_{1}^{*}, \zeta_{2}^{*}, \ldots, \zeta_{n}^{*}\right)
$$

\subsection{Generalized Logarithmic Geometric Operator}

Definition 21. For any collection of $S V N N s \zeta_{p}=\left\langle P_{\xi_{p}}(r), I_{\xi_{p}}(r), N_{\xi_{p}}(r)\right\rangle(p=1, \ldots, n)$ in $\Re$, with $0<\sigma_{p} \leq \min \left\{P_{\xi_{p}}, 1-I_{\xi_{p}}, 1-N_{\xi_{p}}\right\}<1, \sigma \neq 1$. The structure of logarithmic generalized single valued neutrosophic weighted geometric (L-GSVNWG) operator is

$$
L-\operatorname{GSVNWG}\left(\zeta_{1}, \zeta_{2}, \ldots, \zeta_{n}\right)=\left(\sum_{p=1}^{n}\left(\log _{\sigma_{p}}\left(\zeta_{p}\right)^{\gamma}\right)^{\beta_{p}}\right)^{\frac{1}{\gamma}}
$$

where $\beta_{p}(p=1, \ldots, n)$ are weighting vector with $\beta_{p} \geq 0$ and $\sum_{p=1}^{n} \beta_{p}=1$. 
Theorem 6. For any collection of $S V N N s \zeta_{p}=\left\langle P_{\xi_{p}}(r), I_{\xi_{p}}(r), N_{\xi_{p}}(r)\right\rangle(p=1, \ldots, n)$ in $\Re$, with $0<\sigma_{p} \leq$ $\min \left\{P_{\xi_{p}}, 1-I_{\xi_{p}}, 1-N_{\xi_{p}}\right\}<1, \sigma \neq 1, \gamma \geq 1$. Then by using logarithmic operations and definition (21), $L-G S V N W G$ define as

$$
\begin{aligned}
& L-\operatorname{GSVNWG}\left(\zeta_{1}, \zeta_{2}, \ldots, \zeta_{n}\right) \\
& \left\{\begin{array}{c}
\left(1-\left[1-\prod_{p=1}^{n}\left(1-\left(\log _{\sigma_{p}} P_{\xi_{p}}\right)^{\gamma}\right)^{\beta_{p}}\right]^{\frac{1}{\gamma}},\right. \\
\left(1-\prod_{p=1}^{n}\left(1-\left(\log _{\sigma_{p}}\left(1-I_{\xi_{p}}\right)\right)^{\gamma}\right)^{\beta_{p}}\right)^{\frac{1}{\gamma}} \\
\left(1-\prod_{p=1}^{n}\left(1-\left(\log _{\sigma_{p}}\left(1-N_{\xi_{p}}\right)\right)^{\gamma}\right)^{\beta_{p}}\right)^{\frac{1}{\gamma}}
\end{array}\right) \quad 0<\sigma_{p} \leq \min \left\{\begin{array}{c}
P_{\tilde{\xi}_{p},} \\
1-I_{\xi_{p},} \\
1-N_{\xi_{p}}
\end{array}\right\}<1
\end{aligned}
$$

where $\beta_{p}(p=1, \ldots, n)$ is the weighting vector with $\beta_{p} \geq 0$ and $\sum_{p=1}^{n} \beta_{p}=1$.

Apparently, if we use $\gamma=1$, then the $L-G S V N W G$ operator is becomes into $L-S V N W G$ operator.

Proof. Theorem 6 takes the form by utilizing the technique of mathematical induction and the procedure is eliminated here.

Remark 4. If $\sigma_{1}=\sigma_{2}=\sigma_{3}=\ldots=\sigma_{n}=\sigma$, that is $0<\sigma \leq \min \left\{P_{\xi_{p}}, 1-I_{\xi_{p}}, 1-N_{\xi_{p}}\right\}<1, \sigma \neq 1$, then $L-G S V N W G$ operator reduced as follows

$$
L-\operatorname{GSVNWG}\left(\zeta_{1}, \zeta_{2}, \ldots, \zeta_{n}\right)=\left(\begin{array}{c}
1-\left[1-\prod_{p=1}^{n}\left(1-\left(\log _{\sigma} P_{\xi_{p}}\right)^{\gamma}\right)^{\beta_{p}}\right]^{\frac{1}{\gamma}}, \\
\left(1-\prod_{p=1}^{n}\left(1-\left(\log _{\sigma}\left(1-I_{\xi_{p}}\right)\right)^{\gamma}\right)^{\beta_{p}}\right)^{\frac{1}{\gamma}} \\
\left(1-\prod_{p=1}^{n}\left(1-\left(\log _{\sigma}\left(1-N_{\xi_{p}}\right)\right)^{\gamma}\right)^{\beta_{p}}\right)^{\frac{1}{\gamma}}
\end{array}\right) .
$$

\section{Properties}

$L-$ GSVNWG operator satisfies some properties are enlist below;

(1) Idempotency: For any collection of SVNNs $\zeta_{p}=\left\langle P_{\xi_{p}}(r), I_{\xi_{p}}(r), N_{\xi_{p}}(r)\right\rangle(p=1, \ldots, n)$ in $\Re$. Then, if collection of SVNNs $\zeta_{p}=\left\langle P_{\xi_{p}}(r), I_{\xi_{p}}(r), N_{\xi_{p}}(r)\right\rangle(p=1, \ldots, n)$ are identical, that is

$$
L-\operatorname{GSVNWG}\left(\zeta_{1}, \zeta_{2}, \ldots, \zeta_{n}\right)=\zeta \text {. }
$$


(2) Boundedness: for any collection of SVNNs $\zeta_{p}=\left\langle P_{\xi_{p}}(r), I_{\xi_{p}}(r), N_{\xi_{p}}(r)\right\rangle(p=1, \ldots, n)$ in $\Re . \zeta_{p}^{-}=\left\langle\min _{p} P_{\xi_{p}}, \max _{p} I_{\xi_{p}}, \max _{p} N_{\xi_{p}}\right\rangle$ and $\zeta_{p}^{+}=\left\langle\max _{p} P_{\xi_{p}}, \min _{p} I_{\xi_{p}}, \min _{p} N_{\xi_{p}}\right\rangle(p=1, \ldots, n)$ in $\Re$, therefore

$$
\zeta_{p}^{-} \subseteq L-G S V N W G\left(\zeta_{1}, \zeta_{2}, \ldots, \zeta_{n}\right) \subseteq \zeta_{p}^{+} .
$$

(3) Monotonically: for any collection of SVNNs $\zeta_{p}=\left\langle P_{\xi_{p}}(r), I_{\xi_{p}}(r), N_{\xi_{p}}(r)\right\rangle(p=1, \ldots, n)$ in $\Re$. If $\zeta_{p} \subseteq \zeta_{p}^{*}$ for $(p=1, \ldots, n)$, then

$$
L-\operatorname{GSVNWG}\left(\zeta_{1}, \zeta_{2}, \ldots, \zeta_{n}\right) \subseteq L-G S V N W G\left(\zeta_{1}^{*}, \zeta_{2}^{*}, \ldots, \zeta_{n}^{*}\right)
$$

\section{Proposed Technique for Solving Decision-Making Problems}

This section includes the new approach to decision-making based on the single-valued neutrosophic sets, and we will propose a decision-making matrix as indicated below.

Let $H=\left(h_{1}, h_{2}, \ldots, h_{m}\right)$ be a distinct collection of $m$ probable alternatives and $Y=\left(y_{1}, y_{2}, \ldots, y_{n}\right)$ be a finite collection of $n$ criteria, where $h_{i}$ indicate the $i$-th alternatives and $y_{j}$ indicate the $j$-th criteria. Let $D=\left(d_{1}, d_{2}, \ldots, d_{t}\right)$ be a finite set of $t$ experts, where $d_{k}$ indicate the $k$-th expert. The expert $d_{k}$ supply her appraisal of an alternative $h_{i}$ on an attribute $y_{j}$ as a SVNNs $(i=1, \ldots, m ; j=1, \ldots, n)$. The expert's information is represented by the SVNS decision-making matrix $D^{s}=\left[E_{i p}^{(s)}\right]_{m \times n}$. Assume that $\beta_{p}(p=1, \ldots, m)$ is the weight vector of the attribute $y_{j}$, where $0 \leq \beta_{p} \leq 1, \sum_{p=1}^{n} \beta_{p}=1$ and $\psi=\left(\psi_{1}, \psi_{2}, \ldots, \psi_{m}\right)$ be the weights of the decision makers $d_{k}$ such that $\psi_{k} \leq 1, \sum_{k=1}^{n} \psi_{k}=1$.

When we construct the SVNS decision-making matrices, $D^{s}=\left[E_{i p}^{(s)}\right]_{m \times n}$ for decision. Basically, criteria have two types, one is benefit criteria and other one is cost criteria. If the SVNS decision matrices have cost-type criteria metrics $D^{s}=\left[E_{i p}^{s}\right]_{m \times n}$ can be converted into the normalized SVNS

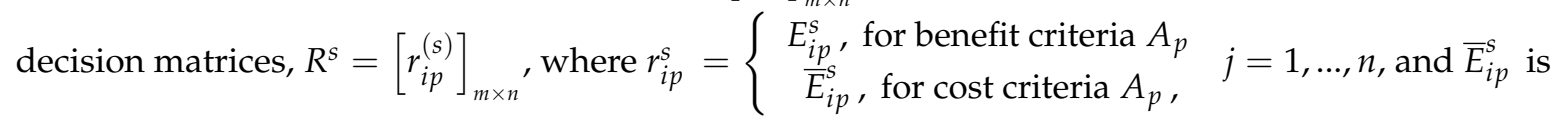
the complement of $E_{i p}^{s}$. The normalization is not required, if the criteria have the same type.

Step 1: In this step, we get the neutrosophic information, using the all proposed logarithmic aggregation operators to evolute the alternative preference values with associated weights, which are $\omega=\left(\omega_{1}, \omega_{2}, \ldots, \omega_{n}\right)$ with $\omega_{p} \geq 0, \Sigma_{p=1}^{n} \omega_{p}=1$.

Step 2: We find the score value $\widetilde{S}\left(\log _{\sigma} \zeta_{p}\right)$ and the accuracy value $\widetilde{A}\left(\log _{\sigma} \zeta_{p}\right)$ of the cumulative total preference value $h_{i}(i=1, \ldots, m)$.

Step 3: By definition, we give ranking to the alternatives $h_{i}(i=1, \ldots, m)$ and choose the best alternative which has the maximum score value.

\subsection{Numerical Example}

Assume that there is a committee which selects five applicable emerging technology enterprises $H_{g}(g=1, \ldots, 5)$, which are given as follows.

(1) Augmented reality $\left(H_{1}\right)$,

(2) Personalized medicine $\left(H_{2}\right)$,

(3) Artificial intelligence $\left(\mathrm{H}_{3}\right)$,

(4) Gene drive $\left(H_{4}\right)$ and

(5) Quantum computing $\left(H_{5}\right)$.

They assess the possible rising technology enterprises according to the five attributes, which are

(1) Advancement $\left(D_{1}\right)$,

(2) Market risk $\left(D_{2}\right)$,

(3) Financial investments $\left(D_{3}\right)$, 
(4) Progress of science and technology $\left(D_{4}\right)$ and

(5) Designs $\left(D_{5}\right)$.

To avoid the conflict between them, the decision makers take the attribute weights as $\beta=(0.15,0.28,0.20,0.22,0.15)^{T}$. They construct the SVNS decision-making matrix given in Table 1 .

Table 1. Emerging Technology Enterprises $D^{1}$.

\begin{tabular}{cccccc}
\hline & $\boldsymbol{D}_{\mathbf{1}}$ & $\boldsymbol{D}_{\mathbf{2}}$ & $\boldsymbol{D}_{\mathbf{3}}$ & $\boldsymbol{D}_{\mathbf{4}}$ & $\boldsymbol{D}_{\mathbf{5}}$ \\
\hline$H_{1}$ & $(0.5,0.3,0.4)$ & $(0.3,0.2,0.5)$ & $(0.2,0.2,0.6)$ & $(0.4,0.2,0.3)$ & $(0.3,0.3,0.4)$ \\
$H_{2}$ & $(0.7,0.1,0.3)$ & $(0.3,0.2,0.7)$ & $(0.6,0.3,0.2)$ & $(0.2,0.4,0.6)$ & $(0.7,0.1,0.2)$ \\
$H_{3}$ & $(0.5,0.3,0.4)$ & $(0.4,0.2,0.6)$ & $(0.6,0.1,0.2)$ & $(0.3,0.1,0.5)$ & $(0.6,0.4,0.3)$ \\
$H_{4}$ & $(0.7,0.3,0.2)$ & $(0.2,0.2,0.7)$ & $(0.4,0.5,0.2)$ & $(0.2,0.2,0.5)$ & $(0.4,0.5,0.4)$ \\
$H_{5}$ & $(0.4,0.1,0.3)$ & $(0.2,0.1,0.5)$ & $(0.4,0.1,0.5)$ & $(0.6,0.3,0.4)$ & $(0.3,0.2,0.4)$ \\
\hline
\end{tabular}

Since $D_{1}, D_{3}$ are benefit-type criteria and $D_{2}, D_{4}$ is cost type criteria, the normalization is required for these decision matrices. Normalized decision matrices are shown in Table 2.

Table 2. Emerging Technology Enterprises $R^{1}$.

\begin{tabular}{cccccc}
\hline & $\boldsymbol{D}_{\mathbf{1}}$ & $\boldsymbol{D}_{\mathbf{2}}$ & $\boldsymbol{D}_{\mathbf{3}}$ & $\boldsymbol{D}_{\mathbf{4}}$ & $\boldsymbol{D}_{\mathbf{5}}$ \\
\hline$H_{1}$ & $(0.5,0.3,0.4)$ & $(0.5,0.2,0.3)$ & $(0.2,0.2,0.6)$ & $(0.3,0.2,0.4)$ & $(0.3,0.3,0.4)$ \\
$H_{2}$ & $(0.7,0.1,0.3)$ & $(0.7,0.2,0.3)$ & $(0.6,0.3,0.2)$ & $(0.6,0.4,0.2)$ & $(0.7,0.1,0.2)$ \\
$H_{3}$ & $(0.5,0.3,0.4)$ & $(0.6,0.2,0.4)$ & $(0.6,0.1,0.2)$ & $(0.5,0.1,0.3)$ & $(0.6,0.4,0.3)$ \\
$H_{4}$ & $(0.7,0.3,0.2)$ & $(0.7,0.2,0.2)$ & $(0.4,0.5,0.2)$ & $(0.5,0.2,0.2)$ & $(0.4,0.5,0.4)$ \\
$H_{5}$ & $(0.4,0.1,0.3)$ & $(0.5,0.1,0.2)$ & $(0.4,0.1,0.5)$ & $(0.4,0.3,0.6)$ & $(0.3,0.2,0.4)$ \\
\hline
\end{tabular}

Step 1: Now, we apply all the proposed logarithmic aggregation operators to collective neutrosophic information as follows.

Case 1: Using logarithmic single-valued neutrosophic hybrid weighted averaging aggregation operator, we obtained the results shown in Table 3.

Table 3. Aggregated information using the logarithmic single valued neutrosophic hybrid weighted averaging (L-SVNHWA) operator for $\sigma=0.3$.

\begin{tabular}{ll}
\hline$H_{1}$ & $(0.17624,0.23432,0.43885)$ \\
$H_{2}$ & $(0.66164,0.16229,0.21840)$ \\
$H_{3}$ & $(0.52788,0.18347,0.32224)$ \\
$H_{4}$ & $(0.49410,0.30962,0.20985)$ \\
$H_{5}$ & $(0.22496,0.12393,0.39318)$ \\
\hline
\end{tabular}

Case 2: Using Logarithmic single valued neutrosophic hybrid weighted geometric aggregation operator, we obtainedthe results shown in Table 4 .

Table 4. Aggregated information using logarithmic single valued neutrosophic hybrid weighted geometric (L-SVNHWG) operator for $\sigma=0.1$.

\begin{tabular}{ll}
\hline$H_{1}$ & $(0.52472,0.12638,0.24189)$ \\
$H_{2}$ & $(0.81968,0.10633,0.11764)$ \\
$H_{3}$ & $(0.74946,0.11782,0.17620)$ \\
$H_{4}$ & $(0.70685,0.18942,0.11685)$ \\
$H_{5}$ & $(0.58497,0.07427,0.23305)$ \\
\hline
\end{tabular}

Step 2: We find the score index $\widetilde{S}\left(\log _{\sigma} \zeta_{p}\right)$ and the accuracy index $\widetilde{A}\left(\log _{\sigma} \zeta_{p}\right)$ of the cumulative overall preference value $h_{i}(i=1,2,3,4,5)$. 
Case 1: Using the score of aggregated information for L-SVNHWA operator, we obtained the results shown in Table 5.

Table 5. Score of aggregated information for L-SVNHWA operator.

\begin{tabular}{cccc}
\hline$\widetilde{S}\left(\log _{0.3} H_{1}\right)$ & -1.14345 & $\widetilde{A}\left(\log _{0.3} H_{1}\right)$ & 0.25985 \\
$\widetilde{S}\left(\log _{0.3} H_{2}\right)$ & 0.30519 & $\widetilde{A}\left(\log _{0.3} H_{2}\right)$ & 1.0087 \\
$\widetilde{S}\left(\log _{0.3} H_{3}\right)$ & -0.02207 & $\widetilde{A}\left(\log _{0.3} H_{3}\right)$ & 0.96078 \\
$\widetilde{S}\left(\log _{0.3} H_{4}\right)$ & -0.08895 & $\widetilde{A}\left(\log _{0.3} H_{4}\right)$ & 0.91781 \\
$\widetilde{S}\left(\log _{0.3} H_{5}\right)$ & -0.76389 & $\widetilde{A}\left(\log _{0.3} H_{5}\right)$ & 0.28571 \\
\hline
\end{tabular}

Case 2: Score of Aggregated information for L-SVNHWG Operator, we obtained the results shown in Table 6.

Table 6. Score of aggregated information for L-SVNHWG operator.

\begin{tabular}{cccc}
\hline$\widetilde{S}\left(\log _{0.1} H_{1}\right)$ & 0.540979 & $\widetilde{A}\left(\log _{0.1} H_{1}\right)$ & 0.89888 \\
$\widetilde{S}\left(\log _{0.1} H_{2}\right)$ & 0.810463 & $\widetilde{A}\left(\log _{0.1} H_{2}\right)$ & 1.01683 \\
$\widetilde{S}\left(\log _{0.1} H_{3}\right)$ & 0.736126 & $\widetilde{A}\left(\log _{0.1} H_{3}\right)$ & 1.01338 \\
$\widetilde{S}\left(\log _{0.1} H_{4}\right)$ & 0.704159 & $\widetilde{A}\left(\log _{0.1} H_{4}\right)$ & 0.994506 \\
$\widetilde{S}\left(\log _{0.1} H_{5}\right)$ & 0.618387 & $\widetilde{A}\left(\log _{0.1} H_{5}\right)$ & 0.903179 \\
\hline
\end{tabular}

Step 3: We find the best (suitable) alternative which has the maximum score value from the set of alternatives $h_{i}(i=1,2,3,4,5)$. Overall preference value and ranking of the alternatives are summarized in Table 7.

Table 7. Overall preference value and ranking of the alternatives.

\begin{tabular}{ccccccc}
\hline & $\widetilde{\boldsymbol{S}}\left(\boldsymbol{H}_{\mathbf{1}}\right)$ & $\widetilde{\boldsymbol{S}}\left(\boldsymbol{H}_{\mathbf{2}}\right)$ & $\widetilde{\boldsymbol{S}}\left(\boldsymbol{H}_{\mathbf{3}}\right)$ & $\widetilde{\boldsymbol{S}}\left(\boldsymbol{H}_{\mathbf{4}}\right)$ & $\widetilde{\boldsymbol{S}}\left(\boldsymbol{H}_{\mathbf{5}}\right)$ & Ranking \\
\hline$L-S V N H W A$ & -1.143 & 0.305 & -0.022 & -0.088 & -0.763 & $\boldsymbol{H}_{2}>H_{3}>H_{4}>H_{5}>H_{1}$ \\
$L-S V N H W G$ & 0.540 & 0.810 & 0.736 & 0.704 & 0.618 & $\boldsymbol{H}_{2}>H_{3}>H_{4}>H_{5}>H_{1}$ \\
\hline
\end{tabular}

\subsection{Comparison with Existing Methods}

This section consists of the comparative analysis of several existing aggregation operators of neutrosophic information with the proposed logarithmic single valued hybrid weighted aggregation operators. Existing methods for aggregated neutrosophic information are shown in Table 8-11.

Table 8. Average aggregated SVN information.

\begin{tabular}{cccc}
\hline & SVNWA [35] & SVNOWA [35] & NWA [14] \\
\hline$H_{1}$ & $(0.3779,0.2259,0.4002)$ & $(0.3820,0.2449,0.4071)$ & $(0.3779,0.2314,0.4223)$ \\
$H_{2}$ & $(0.6615,0.2052,0.2381)$ & $(0.6663,0.1801,0.2430)$ & $(0.6615,0.2426,0.2446)$ \\
$H_{3}$ & $(0.5656,0.1763,0.3131)$ & $(0.5597,0.1838,0.3122)$ & $(0.5656,0.2109,0.3272)$ \\
$H_{4}$ & $(0.5722,0.2929,0.2219)$ & $(0.5706,0.3145,0.2219)$ & $(0.5722,0.3348,0.2338)$ \\
$H_{5}$ & $(0.4165,0.1413,0.3607)$ & $(0.3960,0.1373,0.3696)$ & $(0.4165,0.1633,0.4131)$ \\
\hline
\end{tabular}

Table 9. Average aggregated SVN information.

\begin{tabular}{ccc}
\hline & SVNFWA [12] & SVNHWA [11] $\boldsymbol{\gamma}=\mathbf{2}$ \\
\hline$H_{1}$ & $(0.3755,0.2262,0.4018)$ & $(0.3725,0.2264,0.4033)$ \\
$H_{2}$ & $(0.6611,0.2072,0.2385)$ & $(0.6608,0.2086,0.2388)$ \\
$H_{3}$ & $(0.5652,0.1779,0.3141)$ & $(0.5648,0.1790,0.3149)$ \\
$H_{4}$ & $(0.5692,0.2956,0.2225)$ & $(0.5663,0.2978,0.2230)$ \\
$H_{5}$ & $(0.4159,0.1422,0.3646)$ & $(0.4151,0.1427,0.3680)$ \\
\hline
\end{tabular}


Table 10. Average aggregated SVN information.

\begin{tabular}{ccc}
\hline & SVNHWA [11] $\boldsymbol{\gamma}=\mathbf{3}$ & L-SVNWA [10] \\
\hline$H_{1}$ & $(0.3693,0.2266,0.4048)$ & $(0.3130,0.1753,0.3544)$ \\
$H_{2}$ & $(0.6604,0.2099,0.2390)$ & $(0.6486,0.1989,0.2313)$ \\
$H_{3}$ & $(0.5645,0.1800,0.3157)$ & $(0.4989,0.1733,0.3321)$ \\
$H_{4}$ & $(0.5635,0.3000,0.2234)$ & $(0.5585,0.2736,0.1942)$ \\
$H_{5}$ & $(0.4143,0.1432,0.3714)$ & $(0.2849,0.1249,0.3758)$ \\
\hline
\end{tabular}

Table 11. Average aggregated SVN information.

\begin{tabular}{cc}
\hline & L-SVNOWA [10] \\
\hline$H_{1}$ & $(0.3229,0.1926,0.3607)$ \\
$H_{2}$ & $(0.6549,0.1719,0.2368)$ \\
$H_{3}$ & $(0.4896,0.1823,0.3303)$ \\
$H_{4}$ & $(0.5561,0.2975,0.1942)$ \\
$H_{5}$ & $(0.2442,0.1209,0.3834)$ \\
\hline
\end{tabular}

Now, we analyze the ranking of the alternatives according to their aggregated information (in Table 12).

Table 12. Overall ranking of the alternatives.

\begin{tabular}{cl}
\hline Existing Operators & Ranking \\
\hline NWA [14] & $\boldsymbol{H}_{2}>H_{3}>H_{4}>H_{5}>H_{1}$ \\
SVNWA [35] & $\boldsymbol{H}_{2}>H_{3}>H_{4}>H_{5}>H_{1}$ \\
SVNOWA [35] & $\boldsymbol{H}_{2}>H_{3}>H_{4}>H_{5}>H_{1}$ \\
SVNWG [35] & $\boldsymbol{H}_{2}>H_{3}>H_{4}>H_{5}>H_{1}$ \\
SVNOWG [35] & $\boldsymbol{H}_{2}>H_{3}>H_{4}>H_{5}>H_{1}$ \\
SVNFWA [12] & $\boldsymbol{H}_{2}>H_{3}>H_{4}>H_{5}>H_{1}$ \\
SVNHWA [11] $\gamma=2$ & $\boldsymbol{H}_{2}>H_{3}>H_{4}>H_{5}>H_{1}$ \\
SVNHWA [11] $\gamma=3$ & $\boldsymbol{H}_{2}>H_{3}>H_{4}>H_{5}>H_{1}$ \\
NWG [14] & $\boldsymbol{H}_{2}>H_{3}>H_{4}>H_{5}>H_{1}$ \\
SVNFWG [12] & $\boldsymbol{H}_{2}>H_{3}>H_{4}>H_{5}>H_{1}$ \\
SVNHWG [11] $\gamma=2$ & $\boldsymbol{H}_{2}>H_{3}>H_{4}>H_{5}>H_{1}$ \\
SVNHWG [11] $\gamma=3$ & $\boldsymbol{H}_{2}>H_{3}>H_{4}>H_{5}>H_{1}$ \\
SNWEA [15] & $\boldsymbol{H}_{2}>H_{3}>H_{5}>H_{4}>H_{1}$ \\
L-SVNWA [10] & $\boldsymbol{H}_{2}>H_{4}>H_{3}>H_{5}>H_{1}$ \\
L-SVNOWA [10] & $\boldsymbol{H}_{2}>H_{4}>H_{3}>H_{5}>H_{1}$ \\
L-SVNWG [10] & $\boldsymbol{H}_{2}>H_{4}>H_{3}>H_{1}>H_{5}$ \\
L-SVNOWG [10] & $\boldsymbol{H}_{2}>H_{3}>H_{4}>H_{5}>H_{1}$ \\
\hline Proposed Operators & \multicolumn{1}{c}{ Ranking } \\
\hline L-SVNHWA & $\boldsymbol{H}_{2}>H_{3}>H_{4}>H_{5}>H_{1}$ \\
L-SVNHWG & $\boldsymbol{H}_{2}>H_{3}>H_{4}>H_{5}>H_{1}$ \\
L-GSVNWA & $\boldsymbol{H}_{2}>H_{4}>H_{3}>H_{5}>H_{1}$ \\
L-GSVNWG & $\boldsymbol{H}_{2}>H_{4}>H_{3}>H_{1}>H_{5}$ \\
\hline &
\end{tabular}

The bast alternative was $\mathrm{H}_{2}$. The obtained results utilizing logarithmic single valued neutrosophic hybrid weighted operators and logarithmic generalized single valued neutrosophic weighted operators were same as results shows existing methods. Hence, this study proposed novel logarithmic aggregation operators to aggregate the neutrosophic information more effectively and efficiently. Utilizing the proposed logarithmic aggregation operators, we sound the best alternative from a set of alternatives given by the decision maker. Hence the proposed MCDM technique based on logarithmic operators lets us find the best alternative as an applications in decision support systems. 


\section{Conclusions}

In this work, an attempt has been made to present different kinds of logarithmic weighted averaging and geometric aggregation operators based on the single-valued neutrosophic set environment. Earlier, it has been observed that the various aggregation operators are defined under the SVNSs environment where the aggregation operators based on the algebraic or Einstein $\mathrm{t}$-norm and t-conorm. In this paper, we proposed novel logarithmic hybrid aggregation operators and also logarithmic generalized averaging and geometric aggregation operators. Aggregation operators, namely L-SVNHWA, L-SVNHWG, L-GSVNWA and L-GSVNWA are developed under the SVNSs environment and we have studied their properties in detail. Further, depending on the standardization of the decision matrix and the proposed aggregation operators, a decision-making approach is presented to find the best alternative to the SVNSs environment. An illustrative example is taken for illustrating the developed approach, and their results are compared with some of the existing approaches of the SVNSs environment to show the validity of it. From the studies, we conclude that the proposed approach is more generic and suitable for solving the stated problem.

In the future, we shall link the proposed operators with some novel fuzzy sets, like as type 2 fuzzy sets, neutrosophic sets, and so on. Moreover, we may examine if our constructed approach can also be applied in different areas, such as personal evaluation, medical artificial intelligence, energy management and supplier selection evaluation.

Author Contributions: Conceptualization, S.A. (Shahzaib Ashraf) and S.A. (Saleem Abdullah); methodology, S.A. (Shahzaib Ashraf); software, S.A. (Shahzaib Ashraf); validation, S.A. (Shahzaib Ashraf), S.A. (Saleem Abdullah), F.S. and N.u.A.; formal analysis, F.S. and N.u.A.; investigation, S.A. (Shahzaib Ashraf); writing-original draft preparation, S.A.; writing-review and editing, S.A. (Shahzaib Ashraf); visualization, F.S.; supervision, S.A. (Saleem Abdullah); funding acquisition, F.S.

Funding: This research received no external funding.

Acknowledgments: The authors would like to thank the editor in chief, associate editor and the anonymous referees for detailed and valuable comments which helped to improve this manuscript.

Conflicts of Interest: The authors declare no conflict of interest.

\section{References}

1. Zadeh, L.A. Fuzzy sets. Inf. Control 1965, 8, 338-353. [CrossRef]

2. Atanassov, K.T. Intuitionistic fuzzy sets. Fuzzy Sets Syst. 1986, 20, 87-96. [CrossRef]

3. Smarandache, F. A unifying field in logics: Neutrosophic logic. Am. Res. Press Rehoboth 1999, 1-141.

4. Soria Frisch, A.; Koppen, M.; Sy, T. Is she gonna like it? automated inspection system using fuzzy aggregation. In Intelligent Systems for Information Processing; Elsevier: Amsterdam, The Netherlands, 2003; pp. 465-476.

5. Broumi, S.; Bakal, A.; Talea, M.; Smarandache, F.; Vladareanu, L. Applying Dijkstra algorithm for solving neutrosophic shortest path problem. In Proceedings of the 2016 International Conference on Advanced Mechatronic Systems (ICAMechS), Melbourne, VIC, Australia, 30 November-3 December 2016; IEEE: Piscataway, NJ, USA, 2016; pp. 412-416.

6. Broumi, S.; Bakal, A.; Talea, M.; Smarandache, F.; ALi, M. Shortest path problem under bipolar neutrosphic setting. Appl. Mech. Mater. 2017, 859, 59-66. [CrossRef]

7. Broumi, S.; Bakal, A.; Talea, M.; Smarandache, F.; Vladareanu, L. Computation of shortest path problem in a network with sv-trapezoidal neutrosophic numbers. In Proceedings of the 2016 International Conference on Advanced Mechatronic Systems (ICAMechS), Melbourne, VIC, Australia, 30 November-3 December 2016; IEEE: Piscataway, NJ, USA, 2016; pp. 417-422.

8. Broumi, S.; Smarandache, F. Correlation coefficient of interval neutrosophic set. In Applied Mechanics and Materials; Trans Tech Publications: Zurich, Switzerland, 2013; Volume 436, pp. 511-517. 
9. Broumi, S.; Talea, M.; Smarandache, F.; Bakali, A. Decision-making method based on the interval valued neutrosophic graph. In Proceedings of the Future Technologies Conference (FTC), San Francisco, CA, USA, 6-7 December 2016; pp. 44-50.

10. Garg, H. New logarithmic operational laws and their applications to multiattribute decision making for single-valued neutrosophic numbers. Cognit. Syst. Res. 2018, 52, 931-946. [CrossRef]

11. Liu, P.; Chu, Y.; Li, Y.; Chen, Y. Some generalized neutrosophic number hamacher aggregation operators and their application to group decision making. Int. J. Fuzzy Syst. 2014, 16, 242-255.

12. Nancy, G.H.; Garg, H. Novel single-valued neutrosophic decision making operators under frank norm operations and its application. Int. J. Uncertain. Quant. 2016, 6, 361-375. [CrossRef]

13. Ye, J. Single valued neutrosophic cross-entropy for multicriteria decision making problems. Appl. Math. Model. 2014, 38, 1170-1175. [CrossRef]

14. Ye, J. Subtraction and division operations of simplified neutrosophic sets. Information 2017, 8, 51. [CrossRef]

15. Ye, J. Exponential operations and aggregation operators of interval neutrosophic sets and their decision making methods. SpringerPlus 2016, 5, 1488. [CrossRef] [PubMed]

16. Garg, H.; Nancy, G.H. Multi-Criteria Decision-Making Method Based on Prioritized Muirhead Mean Aggregation Operator under Neutrosophic Set Environment. Symmetry 2018, 10, 280. [CrossRef]

17. Khan, Q.; Hassan, N.; Mahmood, T. Neutrosophic Cubic Power Muirhead Mean Operators with Uncertain Data for Multi-Attribute Decision-Making. Symmetry 2018, 10, 444. [CrossRef]

18. Adlassnig, K.P. Fuzzy set theory in medical diagnosis. IEEE Trans. Syst. Man Cybern. 1986, 16, $260-265$. [CrossRef]

19. Ashraf, S.; Mahmood, T.; Abdullah, S.; Khan, Q. Different Approaches to Multi-Criteria Group Decision Making Problems for Picture Fuzzy Environment. Bull. Braz. Math. Soc. New Ser. 2018, 1-25. [CrossRef]

20. Ashraf, S.; Mehmood, T.; Abdullah, S.; Khan, Q. Picture Fuzzy Linguistic Sets and Their Applications for Multi-Attribute Group. Nucleus 2018, 55, 66-73.

21. Zeng, S.; Asharf, S.; Arif, M.; Abdullah, S. Application of Exponential Jensen Picture Fuzzy Divergence Measure in Multi-Criteria Group Decision Making. Mathematics 2019, 7, 191. [CrossRef]

22. Ashraf, S.; Abdullah, S. Spherical aggregation operators and their application in multi attribute group decision making. Int. J. Intell. Syst. 2019, 34, 493-523. [CrossRef]

23. Ashraf, S.; Abdullah, S.; Mahmood, T.; Ghani, F.; Mahmood, T. Spherical fuzzy sets and their applications in multi-attribute decision making problems. J. Intell. Fuzzy Syst. 2019, 1-16. [CrossRef]

24. Wang, R.; Wang, J.; Gao, H.; Wei, G. Methods for MADM with Picture Fuzzy Muirhead Mean Operators and Their Application for Evaluating the Financial Investment Risk. Symmetry 2019, 11, 6. [CrossRef]

25. Xu, Z. Intuitionistic fuzzy aggregation operators. IEEE Trans. Fuzzy Syst. 2007, 15, 1179-1187.

26. Zhang, H.; Zhang, R.; Huang, H.; Wang, J. Some Picture Fuzzy Dombi Heronian Mean Operators with Their Application to Multi-Attribute Decision-Making. Symmetry 2018, 10, 593. [CrossRef]

27. Ashraf, S.; Abdullah, S.; Mahmood, T. GRA method based on spherical linguistic fuzzy Choquet integral environment and its application in multi attribute decision making problems. Math. Sci. 2018, 12, 263-275. [CrossRef]

28. Cuong, B.C. Picture Fuzzy Sets-First Results. Part 1, Seminar Neuro-Fuzzy Systems with Applications; Technical Report; Institute of Mathematics: Hanoi, Vietnam, 2013.

29. Deschrijver, G.; Cornelis, C.; Kerre, E.E. On the representation of intuitionistic fuzzy t-norms and t-conorms. IEEE Trans. Fuzzy Syst. 2004, 12, 45-61. [CrossRef]

30. Li, L.; Zhang, R.; Wang, J.; Shang, X.; Bai, K. A Novel Approach to Multi-Attribute Group Decision-Making with q-Rung Picture Linguistic Information. Symmetry 2018, 10, 172. [CrossRef]

31. Xu, Z.; Yager, R.R. Some geometric aggregation operators based on intuitionistic fuzzy sets. Int. J. Gen. Syst. 2006, 35, 417-433. [CrossRef]

32. Smarandache, F. Neutrosophy. Neutrosophic Probability, Set, and Logic; ProQuest Information \& Learning: Ann Arbor, MI, USA, 1998.

33. Wang, H.; Smarandache, F.; Zhang, Y.Q.; Sunderraman, R. Single valued neutrosophic sets. Multispace Multistruct. 2010, 4, 410-413. 
34. Zhang, X.; Bo, C.; Smarandache, F.; Dai, J. New inclusion relation of neutrosophic sets with applications and related lattice structure. Int. J. Mach. Learn. Cybern. 2018, 9, 1753-1763. [CrossRef]

35. Peng, J.J.; Wang, J.Q.; Wang, J.; Zhang, H.Y.; Chen, Z.H. Simplified neutrosophic sets and their applications in multi-criteria group decision-making problems. Int. J. Syst. Sci. 2016, 47, 2342-2358. [CrossRef]

(C) 2019 by the authors. Licensee MDPI, Basel, Switzerland. This article is an open access article distributed under the terms and conditions of the Creative Commons Attribution (CC BY) license (http:/ / creativecommons.org/licenses/by/4.0/). 